Ergod. Th. \& Dynam. Sys. (1987), 7, 25-47

Printed in Great Britain

\title{
Topological dynamics and ergodic theory
}

\author{
ROBERT ELLIS \\ School of Mathematics, University of Minnesota, Minneapolis, Minnesota 55455, USA
}

(Received 2 August 1985)

\begin{abstract}
It is shown that when viewed properly some concepts in topological dynamics and ergodic theory are not merely analogous but equivalent. Also the Mackey-Halmos-von Neumann theorem on ergodic processes with discrete spectrum is generalized and an account of the Mackey-Zimmer theory of minimal cocycles is given in a more general setting.
\end{abstract}

\section{Introduction}

This paper is an attempt to link topological dynamics and ergodic theory more closely. There are many concepts which have counterparts in both theories, e.g., minimality-ergodicity, equicontinuous-discrete spectrum, almost periodic extension-relatively discrete spectrum, etc. These have led to the formulation of analogous theorems. However, they have up until now remained analogies; the proofs involved being entirely different and neither directly deducible from the other. This, of course, is not surprising since the methods used in one are topological and in the other measure theoretic.

However, there is a meeting ground. Thus, let $(X, \mu)$ be a 'probability space' and $T$ a group of measure preserving transformations on $X$. Then $T$ acts as a group of homeomorphisms on the Gelfand space $U$ of $L^{\infty}(X, \mu)$. Now $\mu$ induces a $T$ invariant probability measure $\nu$ on $U$ such that $L^{\infty}(U, \nu) \cong L^{\infty}(X, \mu)$ and since ergodic theory is 'really' about the measure algebra on $X$ and not the flow $(X, T)$ itself, $(U, T)$ captures all the ergodic-theoretic essentials. Consequentially, there is hope that some ergodic theorems could be deduced by topological means from the flow $(U, T, \nu)$. (As a simple example it is immediate that $(X, T, \mu)$ is ergodic if and only if $(U, T)$ is topologically transitive.)

A priori there are problems with this approach. Usually the group $T$ is provided with a topology such that the action of $T$ on $X$ is measurable and this does not entail the continuity of the map $(u, t) \rightarrow u t: U \times T \rightarrow U$. However, as with most problems in topological dynamics this is a mere technicality and one may assume without loss of generality that $T$ is discrete. (An example of how the given topology on $T$ may be introduced at the 'critical' moment is given in $\S 3$.)

Another problem is that the space $U$ is 'huge' and that $(U, T)$ is in general not minimal, though in the situations discussed it is topologically transitive. This is a real drawback since there is a rather extensive theory for minimal flows which has 
as yet not been extended to the topologically transitive case. (One approach might be through homomorphic images and universe limits. Recall that a distal, topologically transitive flow is minimal, [5]).

At this point it should also be mentioned that nowhere in this paper is it assumed that $(X, \mu)$ is a standard Borel space. This assumption seems to be made for technical reasons, but is unnecessary for the results contained herein. (Thus, the latter are generalizations of those in Zimmer [10] which are in turn generalizations of the classical results.) One of the principal uses of this assumption is to produce a disintegration of $\mu$ with respect to $\rho$ when there is a homomorphism of the system $(X, T, \mu)$ onto $(Y, T, \rho)$. In our context this is replaced by the conditional expectation. (See $\$ 2$ for details.)

The disadvantages mentioned above are offset by some useful properties of the space $U$. These include:

(i) $L^{\infty}(U) \cong C(U)$ and so one may always use continuous functions, thus avoiding arguments involving sets of measure zero;

(ii) $(U, \mu)$ is a hyperstone space (see [2]) which means that a subset $A$ of $U$ has measure 0 if and only if it is of the first category;

(iii) $U$ is extremely disconnected and as such has many useful topological properties (see [2] and [6]) which are exploited throughout this paper.

As in [4] it is more convenient to look at $C(X)$ rather than $X$. Also, even though the flows involved are not minimal and there is in general no universal object, it is still convenient to work in the category of $T$-subalgebras of a given algebra, $U$. The basic situation is described in $\S 1$.

The introductions to the various sections discuss the relation between the dynamical and ergodic notions studied in that section. Thus, the reader might find it helpful to read these before plunging into the 'gory' details.

At this point suffice it to say that $\S 2$ deals with disjointness-independence, $\S 3$ with equicontinuity-discrete spectrum, $\S 4$ with the Mackey theory of minimal cocycles and ergodic decompositions, and $\S 5$ with the twin notions almost periodic extension-relatively discrete spectrum.

Much of the material of this paper is considered from a different perspective in Zimmer [10]. Indeed, the point of view presented here developed in part as an attempt to understand Zimmer's paper.

\section{Basic Material}

In this section the underlying situation discussed in this paper is described. Good references for this material are [1] and [2].

(1.1) Standing Notation. Throughout this paper $U$ will denote a fixed compact Hausdorff space, $U$ the algebra of complex valued continuous functions on $U$, and $m$ a fixed regular supported Borel probability measure on $U$. It is also assumed that given $f \in L^{\infty}(\tilde{U})$ there exists $g \in \mathscr{U}$ such that $f=g$ (a.e m).

(1.2) Remarks. (a) The pair $(U, m)$ constitutes a hyperstone space in the terminology of [2]. 
(b) Let $(X, \mu)$ be a probability space and $U$ the Gelfand space of $L^{\infty}(X)$. Then $\mu$ induces a measure $m$ on $U$ such that the pair $(U, m)$ has the properties required in (1.1).

(c) Indeed, the space $U$ of (1.1) may be identified with the Gelfand space of $L^{\infty}(U)$

(1.3) Notation. The topologies of uniform convergence and convergence in measure on $U$ will be denoted by $\mathscr{T}_{u}$ and $\mathscr{T}_{m}$ respectively. If $\mathscr{A} \subset \mathcal{U}$, then $\overline{\mathscr{A}}(\tilde{\mathscr{A}})$ will denote its closure with respect to $\mathscr{T}_{u}\left(\mathscr{T}_{m}\right)$.

The following is well known. (See [1].)

(1.4) TheOREM. (i) $\mathscr{T}_{m} \subset \mathscr{T}_{u}$.

(ii) If the sequence $\left(f_{n}\right)$ converges to $f$ (a.e.), then it converges to $f$ in $\mathscr{T}_{m}$.

(iii) If the sequence $\left(f_{n}\right)$ converges to $f$ in $\mathscr{T}_{m}$ then there exists a subsequence $\left(f_{n_{i}}\right)$ of $\left(f_{n}\right)$ such that $f_{n_{i}} \rightarrow f$ (a.e.).

(iv) Both $\mathscr{T}_{m}$ and $\mathscr{T}_{u}$ are metrizable.

(1.5) Definition. The subset $\mathscr{A}$ of $U$ is a subalgebra of $U$ if it is a subspace of $U$ containing the constant function 1 and is closed under multiplication and complex conjugation.

Let $\mathscr{A} \subset \mathcal{U}$. Then $R(\mathscr{A})$ will denote the equivalence relation on $U: x \equiv y(R(\mathscr{A}))$ if $f(x)=f(y)(f \in \mathscr{A})$, and $|\mathscr{A}|$ will denote the quotient space $U / R(\mathscr{A})$.

It is well known that $|\mathscr{A}|$ is a compact Hausdorff space and that $m_{\mathscr{A}}=m \Pi_{\mathscr{A}}^{-1}$ is a supported probability measure on $|\mathscr{A}|$. (Here $\Pi_{\mathscr{A}}$ is the canonical map of $U$ onto $|\mathscr{A}|$.) When there is no danger of confusion $m_{\mathscr{A}}$ will also be denoted by $m$.

(1.6) TheOREM. Let $\mathscr{A}$ be a subalgebra of $\mathcal{U}$ and $\Pi: U \rightarrow|\mathscr{A}|$ the canonical map. Then

(i) $\Pi^{*} C(|\mathscr{A}|)=\bar{A}$; and

(ii) $\Pi^{*} L^{\infty}(|\mathscr{A}|)=\tilde{A}$.

Proof. (i) Clearly $\Pi^{*} C(|\mathscr{A}|)$ is a uniformly closed subset of $\mathscr{U}$ containing $\mathscr{A}$, whence $\bar{A} \subset \Pi^{*} C(|\mathscr{A}|)$. On the other hand every element $f$ of $\bar{A}$ induces $\hat{f} \in C(|\mathscr{A}|)$ with $\Pi^{*}(f)=f \circ \Pi=\hat{f}$. Moreover, the set $\mathscr{B}=\{\hat{f} \mid f \in \bar{A}\}$ is a uniformly closed subalgebra of $C(|\mathscr{A}|)$ which separates points. Thus, $\mathscr{B}=C(|\mathscr{A}|)$, whence $\Pi^{*}(|\mathscr{A}|) \subset \bar{A}$.

(ii) Let $f \in L^{\infty}(|\mathscr{A}|)$. Then there exists a sequence $\left(f_{n}\right)$ in $C(|\mathscr{A}|)$ which converges to $f$ (a.e. $m \Pi^{-1}$ ). Hence, $\left(f_{n} \circ \Pi\right)$ converges to $f \circ \Pi$ (a.e. $m$ ) and so

$$
\Pi^{*} f=f \circ \Pi \in \operatorname{cls}_{\mathscr{T}_{m}} \overline{\mathscr{A}}=\tilde{\mathscr{A}} \text {. }
$$

On the other hand let $f \circ \Pi \in \tilde{\mathscr{A}}$. Then by (1.4) there exists a sequence $\left(g_{n}\right)$ contained in $\mathscr{A}$ which converges to $f \circ \Pi$ (a.e. $m$ ).

Now $g_{n}$ induces $f_{n} \in C(|\mathscr{A}|)$ with $g_{n}=f_{n} \circ \Pi$ for all $n$. Set $K=\left\{x \in|\mathscr{A}|: f_{n}(x) \rightarrow f(x)\right\}$ and $L=\left\{u \in U \mid g_{n}(u) \rightarrow f(\Pi(u))\right\}$. Then $\Pi^{-1}(|\mathscr{A}| \backslash K) \subset U \backslash L$ and $m(U \backslash L)=0$ implies that $m \Pi^{-1}(|\mathscr{A}| \backslash E)=0$. Consequently $f \in L^{\infty}(|\mathscr{A}|)$.

(1.7) Remarks. Let $\mathscr{A}$ be a subalgebra of $\mathscr{U}$ with $\mathscr{A}=\tilde{A}$. Then (1.6) implies that the pair $(|\tilde{A}|, m)$ is a hyperstone space and $m$ is a normal or category measure on $|\tilde{A}|$ [2]. Among other things this implies that a Borel subset $A$ of $|\tilde{\mathscr{A}}|$ is of the first category if and only if $m(A)=m(\bar{A})=0$. Moreover the space $|\tilde{A}|$ is extremely 
disconnected, i.e. the closure of an open subset of $|\tilde{\mathscr{A}}|$ is again open, hence both open and closed.

(1.8) ThEOREM. Let $\mathscr{F}, \mathscr{A}$ be subalgebras of $\mathscr{U}$ with $\mathscr{F}=\tilde{\mathscr{F}} \subset \mathscr{A}=\tilde{\mathscr{A}}$. Then the canonical map of $|\mathscr{A}|$ onto $|\mathscr{F}|$ is open.

Proof. Let $N$ be a non-vacuous subset of $|\mathscr{A}|$ which is both open and closed. If the interior of the closed subset $\Pi(N)$ were null, $\Pi(N)$ would be of the first category, whence by (1.7)

$$
0=m_{\mathscr{F}}(\Pi(N))=m_{\mathscr{A}}(N) .
$$

But this is impossible since $m_{s}$ is supported.

Now let $M=\mathrm{cls}$ int $\Pi(N)$. Then $M$ is open (1.7) and $M \subset \Pi(N)$. Since $N \backslash \Pi^{-1}(M)$ is both open and closed, and int $\left(\Pi\left(N \backslash \Pi^{-1}(M)\right)=\varnothing\right.$, $N \backslash \Pi^{-1}(M)=\varnothing$. Hence $\Pi(N)=M$ which is open. The theorem now follows from (1.7).

(1.9) COROllaRy. Let $\mathscr{F}, \mathscr{A}$ be subalgebras of $U$ with $\mathscr{F}=\tilde{\mathscr{F}} \subset \mathscr{A}$. Then the canonical map of $|\mathscr{A}|$ onto $|\mathscr{F}|$ is open.

Proof. This follows from (1.8) and the fact that

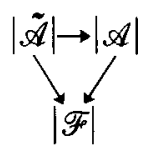

is commutative, where the arrows are the canonical maps.

\section{Independence and disjointness}

One of the most useful tools in ergodic theory is the theorem on the disintegration of measures. Since in general this requires some sort of countability assumption, it is unavailable to us. The device which replaces it is the conditional expectation which yields a continuous disintegration in the proper context; (see (2.3)).

Consideration of conditional expectation leads naturally to the notion of independence and its topological counterpart, disjointness; (see definition (2.8)). Theorem (2.10) relates these two concepts.

(2.1) Definition (Conditional expectation). Let $\mathscr{A}$ be a subalgebra of $\mathcal{U}$. Then $E_{\mathscr{A}}: \mathcal{U} \rightarrow \tilde{\mathscr{A}}$ is the map such that

$$
m(f g)=m_{\tilde{\mathscr{A}}}\left(\left(E_{\mathscr{A}} f\right) g\right) \quad(f \in \mathcal{U}, g \in \tilde{A}) .
$$

The existence of $E_{\mathscr{A}}$ and the properties given below are standard results.

(2.2) Remarks. Let $\mathscr{A}, \mathscr{B}$ be subalgebras of $\mathcal{U}$ with $\mathscr{A} \subset \mathscr{B}$. Then

(1) $E_{\mathscr{A}}$ is linear.

(2) $E_{\mathscr{A}} f=f \quad(f \in \tilde{\mathscr{A}})$.

(3) $E_{\mathscr{A}} E_{\mathscr{B}}=E_{\mathscr{B}}$.

(4) $E_{\mathscr{A}}(f g)=f E_{\mathscr{A}}(g) \quad(f \in \tilde{A}, g \in \mathcal{U})$.

(5) $E_{\mathscr{A}} f \geq 0 \quad(f \in \mathcal{U}, f \geq 0)$. 
(2.3) THEOREM. Let $\mathscr{A}, \mathscr{B}$ be subalgebras of $\mathscr{U}$ with $\mathscr{A} \subset \mathscr{B}$ and let $\lambda_{y}$ be the map $f \rightarrow\left(E_{\mathscr{A}} f\right)(y): \tilde{\mathscr{B}} \rightarrow \mathbb{C}(y \in|\tilde{A}|)$. Then:

(1) $\lambda_{y} \in M(|\tilde{\mathscr{B}}|) \quad(y \in|\hat{\mathscr{A}}|)$.

(2) $\operatorname{supp} \lambda_{y} \subset|\tilde{\mathscr{B}}|_{y}=\{x \in|\tilde{\mathscr{B}}|: x \tilde{\mathscr{A}}=y\} \quad(y \in|\tilde{\mathscr{A}}|)$.

(3) The map $y \stackrel{\wedge}{\rightarrow} \lambda_{y}:|\tilde{A}| \rightarrow M(|\tilde{\mathscr{B}}|)$ is continuous.

(4) $\int_{|\tilde{B}|} f d m=\int_{|\tilde{\mathscr{A}}|} \lambda_{y}(f) d m(y), \quad(f \in \tilde{\mathscr{B}})$.

Proof. (1) This follows from (1) and (5) of (2.2) and the fact that $E_{\mathscr{A}}(1)=1$.

(2) Let $\Pi:|\tilde{B}| \rightarrow|\tilde{A}|$ be the canonical map and suppose $x \notin \Pi^{-1}(y)$. Then there exists an open-closed neighbourhood $N$ of $y$ with $x \notin \Pi^{-1}(N)$.

Let $g$ be the characteristic function of $N$ and $f=1-g$. Then $f \in \tilde{A}$ and so $f=E_{\mathscr{A}}(f)$. Hence

$$
\lambda_{y}(f)=\left(E_{\mathscr{A}} f\right)(y)=f(y)=0
$$

i.e. $\lambda_{y}\left(|\tilde{\mathscr{B}}| \backslash \Pi^{-1}(N)\right)=0$.

(3) Let $\left(y_{\alpha}\right)$ be a net in $|\tilde{\mathscr{A}}|$ which converges to $y \in|\tilde{\mathscr{A}}|$ and let $f \in \tilde{\mathscr{B}}$. Then

$$
\lambda_{y}(f)=\left(E_{\mathscr{A}} f\right)(y)=\lim E_{\mathscr{A}} f\left(y_{\alpha}\right)=\lim \lambda_{y_{\alpha}}(f),
$$

since $E_{\mathscr{A}} f$ is a continuous function.

(4) Set $g=1$ in the equation which defines $E_{\& A}$.

(2.4) Lemma. Let $\mathscr{F}=\tilde{F}, \mathscr{A}=\tilde{\mathscr{A}}$ be subalgebras of $U$ with $\mathscr{F} \subset \mathscr{A}, N$ a non-vacuous open-closed subset of $|\mathscr{A}|, f$ the characteristic function of $N, g=E_{\mathscr{F}} f$, and $\Pi:|\mathscr{A}| \rightarrow|\mathscr{F}|$ the canonical map. Then:

(1) $g(y)=0 \quad(y \notin \Pi(N))$; and

(2) $g^{-1}(0, \infty)$ is dense in $\Pi(N)$.

Proof. (1) Let $h$ be the characteristic function of the open-closed subset $\Pi(N)^{\prime}$ of $|\mathscr{F}|$. Then

$$
\int_{\Pi(N)^{\prime}} g=\int_{|\mathscr{F}|} h g=\int_{|\mathscr{F}|} h E_{\mathscr{F}}(f)=\int_{|\mathscr{A}|}(h \circ \Pi) f=0 .
$$

(2) By $(1), \overline{g^{-1}(0, \infty)}$ is an open-closed subset of $\Pi(N)$. Hence $W=$ $\Pi(N) \backslash \overline{g^{-1}(0, \infty)}$ is again both open and closed. Since $g$ vanishes on $W$,

$$
0=\int_{|\mathscr{F}|} g \chi_{w}=\int_{|\mathscr{F}|} E(f) \chi_{w}=\int_{|\mathscr{A}|} f \chi_{w} \circ \Pi=m_{\mathscr{A}}\left(N \cap \Pi^{-1}(W)\right) .
$$

If $W$ were not null, then $\Pi(n) \in W$ for some $n \in N$. Consequently $N \cap \Pi^{-1}(W)$ would be a non-vacuous open set. But then $m_{\mathscr{A}}\left(N \cap \Pi^{-1}(W)\right) \neq 0$. The proof is completed.

(2.5) THEOREM. Let $\mathscr{A}=\tilde{\mathscr{A}}, \mathscr{B}=\tilde{\mathscr{B}}$ be subalgebras of $U, \mathscr{F}=\mathscr{A} \cap \mathscr{B}, \lambda:|\mathscr{F}| \rightarrow|\mathscr{A}|$, $\mu:|\mathscr{F}| \rightarrow|\mathscr{B}|$ as in (2.3). Then:

(1) $y \rightarrow \rho(f) \lambda_{y} \times \mu_{y}(f):|\mathscr{F}| \rightarrow \mathbb{R}$ is continuous $(f \in C(|\mathscr{A}| \times|\mathscr{B}|, \mathbb{R}))$.

(2) The map $f \rightarrow \int_{|\mathscr{F}|} \lambda_{y} \times \mu_{y}(f) d m(y): C(|\mathscr{A}| \times|\mathscr{B}|, \mathbb{R}) \rightarrow \mathbb{B}$ is positive and linear and so determines a measure $m_{\mathscr{A}} \otimes m_{\mathscr{B}}$ on $|\mathscr{A}| \times|\mathscr{B}|$.

(3) $m_{\mathscr{A}} \otimes m_{\mathscr{B}}(|\mathscr{A}| \times|\mathscr{B}|)=1$ and $\operatorname{supp} m_{\mathscr{A}} \otimes m_{\mathscr{A}}=|\mathscr{A}| \times_{\mathscr{F}}|\mathscr{B}|=\{(x, y): x \in|\mathscr{A}|, y \in$ $|\mathscr{B}|, x \mathscr{F}=y \mathscr{F}\}$, the so called fibred product of $|\mathscr{A}|$ and $|\mathscr{B}|$ over $|\mathscr{F}|$. (Notice that $\mathscr{F}=\tilde{F}$.) (Here $x \mathscr{F}$ denotes the image of $x$ in $|\mathscr{F}|$ under the canonical map.) 
Proof. (1) Let $h \in \mathscr{A}=C(|\mathscr{A}|), g \in \mathscr{B}=C(|\mathscr{B}|)$, and $h \times g$ the map $(x, y) \rightarrow$ $h(x) g(y):|\mathscr{A}| \times|\mathscr{B}| \rightarrow \mathbb{R}$. Then $\rho(h \times g)$ is the map $y \rightarrow \lambda_{y}(h) \mu_{y}(g):|\mathscr{F}| \rightarrow \mathbb{R}$ which is continuous by (2.3).

Since every element of $C(|\mathscr{A}| \times|\mathscr{B}|)$ is the uniform limit of linear combinations of elements of the form $h \times g$ and all the measures $\lambda_{y} \times \mu_{y}$ are of norm 1, statement (1) follows.

(2) This follows immediately from the definition of $\lambda_{y}, \mu_{y}(y \in|\mathscr{F}|)$

(3) That $m_{\mathscr{A}} \otimes m_{\mathscr{B}}(|\mathscr{A}| \times|\mathscr{B}|)=1$ is clear. Let $x \in|\mathscr{A}|, y \in|\mathscr{B}|$ with $(x, y) \not$ $|\mathscr{A}| \times{ }_{\mathscr{F}}|\mathscr{B}|$. Choose open-closed neighbourhoods $M$ and $N$ of $x$ and $y$ respectively such that

$$
M \times N \cap|\mathscr{A}| \times|\mathscr{B}|=\varnothing,
$$

and let $g=E_{\mathscr{F} X}, h=E_{\mathscr{F}} \chi_{N}$. Then

$$
\Pi_{1}(M) \cap \Pi_{2}(N)=\varnothing
$$

where $\Pi_{1}:|\mathscr{A}| \rightarrow|\mathscr{F}|$ and $\Pi_{2}:|\mathscr{B}| \rightarrow|\mathscr{F}|$ are the canonical maps. Consequently

$$
\lambda_{z} \times \mu_{z}(M \times N)=\lambda_{z}(M) \mu_{z}(N)=g(z) h(z)=0 \quad(z \in|\mathscr{F}|)
$$

by lemma (2.4). Hence $m_{\mathscr{A}} \otimes m_{\mathscr{A}}(M \times N)=0$ and so $(x, y) \notin \operatorname{supp} m_{\mathscr{A}} \otimes m_{\mathscr{B}}$.

Now let $(x, y) \in|\mathscr{A}| \times_{\mathscr{F}}|\mathscr{B}|, M, N$ be open-closed neighbourhoods of $x$ and $y$ respectively, and let $\Pi_{1}, \Pi_{2}, g$, and $h$ be as defined above. This time $\Pi_{1}(x)=\Pi_{2}(y)$ and so $\Pi_{1}(M) \cap \Pi_{2}(N)$ is a non-vacuous open-closed subset of $|\mathscr{F}|$. Consequently by (2) of lemma (2.4), $g h$ is a nowhere negative continuous function on $|\mathscr{F}|$ which does not vanish identically. Then

$$
m_{\mathscr{A}} \otimes m_{\mathscr{B}}(M \times N)=\int_{|\mathscr{F}|} g h d m_{\mathscr{F}}>0 .
$$

The proof is completed.

(2.6) THEOREM. With the same assumptions as in (2.5)

$$
E(f \times g)=E_{\mathscr{F}}(f) E_{\mathscr{F}}(g) \quad(f \in \mathscr{A}, g \in \mathscr{B}),
$$

where $E$ is the expectation operator induced by the canonical map, $r$ of $(|\mathscr{A}| \times \mathscr{F}|\mathscr{B}|$, $\left.m_{\mathscr{A}} \otimes m_{\mathscr{B}}\right)$ onto $\left(|\mathscr{F}|, m_{\mathscr{F}}\right)$.

Proof. It suffices to take the case $f=\chi_{A}$ and $g=\chi_{B}$ where $A$ and $B$ are open-closed subsets of $|\mathscr{A}|$ and $|\mathscr{B}|$ respectively.

Let $J=\int_{|\mathscr{A}| \times \mathscr{F}|\mathscr{B}|}(f \times g) \varphi \circ r$. Then we must show that $J=\int_{|\mathscr{F}|} F G \varphi, \varphi \in \mathscr{F}$, where $F=E_{\mathscr{F}}(f)$ and $G=E_{\mathscr{F}}(g)$.

Again we may assume $\varphi=\chi_{N}$ where $N$ is an open-closed subset of $|\mathscr{F}|$. Then

$$
r^{-1}(N)=\Pi_{1}^{-1}(N) \times \Pi_{2}^{-1}(N) \cap|\mathscr{A}| \times_{\mathscr{F}}|\mathscr{B}|
$$

and $(f \times g)(\varphi \circ r)$ equals the characteristic function of $C \times D \cap|\mathscr{A}| \times_{\mathscr{F}}|\mathscr{B}|$ where $C=\Pi_{1}^{-1}(N) \cap A$ and $D=\Pi_{2}^{-1}(N) \cap B$. (Here $\Pi_{1}, \Pi_{2}$ are the canonical maps of $|\mathscr{A}|$, $|\mathscr{B}|$ onto $|\mathscr{F}|$ respectively.) Then

$$
J=m_{\mathscr{A} \otimes \mathscr{B}}(C \times D)=\int_{|\mathscr{F}|} \lambda_{z} \times \mu_{z}(C \times D) d m_{\mathscr{F}}(z)=\int_{|\mathscr{F}|} \lambda_{z}(C) \mu_{z}(D) d m_{\mathscr{F}}(z)
$$

since supp $m_{\mathscr{A} \otimes \mathscr{B}} \subset|\mathscr{A}| \times_{\mathscr{F}}|\mathscr{B}|$, (by (3) of (2.5)). 
Now

and

$$
\lambda_{z}(C)=\left(E_{\mathscr{F} \chi}\right)(z)=E_{\mathscr{F}}\left(\left(\chi_{N} \circ \Pi_{1}\right) \chi_{A}\right)(z)=\chi_{N}(z) F(z)
$$

$$
\mu_{z}(D)=\chi_{N}(z) G(z) .
$$

Thus $J=\int_{|\mathscr{F}|} F G \varphi$. The proof is completed.

(2.7) ThEOREM. With the same notation as in (2.6), let $\kappa$ be the map $u \rightarrow$ $(u \mathscr{A}, u \mathscr{B}): U \rightarrow|\mathscr{A}| \times|\mathscr{B}|$. Then:

(1) $\kappa(U) \subset|\mathscr{A}| \times_{\mathscr{F}}|\mathscr{B}|$; and

(2) $E_{\mathscr{F}}(f g)=E_{\mathscr{H}}(f) E_{\mathscr{H}}(g) \quad(f \in \mathscr{A}, g \in \mathscr{B})$ if and only if $\kappa$ is onto and $m \kappa^{-1}=$ $m_{\mathscr{A}} \otimes_{\mathscr{F}} m_{\mathscr{B}}$.

Proof. (1) This follows directly from the definitions of $\kappa$ and $|\mathscr{A}| \times_{\mathscr{F}}|\mathscr{B}|$.

(2) Necessity. Let $(x, y) \in|\mathscr{A}| \times_{\mathbb{F}}|\mathscr{B}|$ and suppose it is not in $\kappa(U)$. Since the latter is closed, there exist open-closed neighbourhoods $M$ and $N$ of $x$ and $y$ respectively with $M \times N \cap \kappa(U)=\varnothing$. Let $g=E_{\mathscr{F}}\left(\chi_{M}\right)$ and $h=E_{\mathscr{F}}\left(\chi_{N}\right)$. Then by (2) of (2.4), $g^{-1}(0, \infty)$ is dense in $p(M)$ and $h^{-1}(0, \infty)$ is dense in $q(N)$ where $p$ and $q$ are the canonical maps of $|\mathscr{A}|$ and $|\mathscr{B}|$ onto $|\mathscr{F}|$ respectively. Since $(x, y) \in|\mathscr{A}| \times_{\mathscr{F}}|\mathscr{B}|$, this implies that $p(M) \cap q(N)$ is a non-vacuous open subset of $|\mathscr{F}|$ whence $g h \neq 0$. On the other hand $M \times N \cap \kappa(U)=\varnothing$ implies that $\left(\chi_{M} \circ \kappa\right)\left(\chi_{N} \circ \kappa\right)=0$ whence $0=$ $E_{\mathscr{F}}\left(\chi_{M} \chi_{N}\right) \neq g h=E_{\mathscr{F}}\left(\chi_{M}\right) E_{\mathscr{F}}\left(\chi_{N}\right)$, a contradiction. Thus $\kappa$ is onto.

Now let $A$ and $B$ be open-closed subsets of $|\mathscr{A}|$ and $|\mathscr{B}|$ respectively. Then

$$
\begin{aligned}
m_{\mathscr{A}} \otimes m_{\mathscr{B}}(A \times B) & =\int_{|\mathscr{F}|} E_{\mathscr{F}}\left(\chi_{A}\right) E_{\mathscr{F}}\left(\chi_{B}\right) d m_{\mathscr{F}} \\
& =\int_{|\mathscr{F}|} E_{\mathscr{F}}\left(\chi_{A} \chi_{B} \circ \kappa\right) d m_{\mathscr{F}} \\
& =\int_{U}\left(\chi_{A} \chi_{B} \circ \kappa\right) d m .
\end{aligned}
$$

Since $\kappa(u)=|\mathscr{A}| \times_{\mathscr{F}}|\mathscr{B}|, \chi_{A} \chi_{B}{ }^{\circ} \kappa=\chi_{L}$ where $L=\kappa^{-1}\left(A \times B \cap|\mathscr{A}| \times_{\mathscr{F}}|\mathscr{B}|\right)$. Hence

$$
m_{\mathscr{A}} \otimes m_{\mathscr{B}}(A \times B)=m \kappa^{-1}(A \times B) \text {. }
$$

The proof of necessity is complete.

Sufficiency follows from the fact that if $\kappa$ is onto and $m_{\mathscr{A}} \otimes_{\mathscr{F}} m_{\mathscr{B}}=m \kappa^{-1}$, then $E_{\mathscr{F}}(f g)=E_{\mathscr{F}}(f) E_{\mathscr{F}}(g)$ by (2.6) for

$$
(f \circ \kappa)(g \circ \kappa)=f \times\left. g\right|_{|\mathscr{A}| \times \mathscr{F}|\mathscr{B}|}, \quad(f \in \mathscr{A}, g \in \mathscr{B}) .
$$

(2.8) Definition. Let $\mathscr{F}$ be a subalgebra of $\mathcal{U}$ and $f, g \in \mathcal{U}$. Then $f$ and $g$ are independent over $\mathscr{F}$ if $E_{\mathscr{F}}(f g)=E_{\mathscr{F}}(f) E_{\mathscr{F}}(g)$. The algebras $\mathscr{A}$ and $\mathscr{B}$ are independent over $\mathscr{F}$ if $f$ and $g$ are independent over $\mathscr{F}(f \in \mathscr{A}, g \in \mathscr{B})$.

The algebras $\mathscr{A}$ and $\mathscr{B}$ are disjoint over $\mathscr{F}$ if $\mathscr{F} \subset \mathscr{A} \cap \mathscr{B}$ and $\kappa(U)=|\mathscr{A}| \times \mathscr{F}|\mathscr{B}|$ where $\kappa$ is the map $u \rightarrow(u \mathscr{A}, u \mathscr{B}): U \rightarrow|\mathscr{A}| \times|\mathscr{B}|$.

The definitions coincide with the usual ones.

(2.9) THEOREM. Let $\mathscr{A}, \mathscr{B}$, and $\mathscr{F}$ be subalgebras of $\mathcal{U}$. Then:

(1) $\mathscr{A}$ and $\mathscr{B}$ are independent over $\mathscr{F}$ if and only if $\tilde{\mathscr{A}}$ and $\tilde{\mathscr{B}}$ are independent over $\tilde{F} ;$ and 
(2) If $\mathscr{A}$ and $\mathscr{B}$ are independent over $\mathscr{F}$, then $\mathscr{A} \vee \mathscr{F}$ and $\mathscr{B} \vee \mathscr{F}$ are also independent over $\mathscr{F}$.

Proof. (1) Necessity. Let $f \in \tilde{\mathscr{A}}$ and $g \in \mathscr{B}$. Then there exists a sequence $\left(f_{n}\right) \subset \mathscr{A}$ which converges to $f$ in measure. By choosing a subsequence we may suppose that $\left(f_{n}\right)$ converges to $f$ a.e. Furthermore since $f$ is bounded, we may assume that $\left(f_{n}\right)$ is uniformly bounded. Then Lebesgue's bounded convergence theorem implies that $E_{\mathscr{F}}\left(f_{n}\right)$ converges to $E_{\mathscr{F}}(f)$, whence $E_{\mathscr{F}}\left(f_{n}\right) E_{\mathscr{F}}(g)=E_{\mathscr{F}}\left(f_{n} g\right)$ converges to $E_{\mathscr{F}}(f g)$. Thus $\tilde{\mathscr{A}}$ and $\mathscr{B}$ are independent over $\tilde{\mathscr{F}}\left(E_{\mathscr{F}}=E_{\tilde{F}}\right)$. Another application of this argument shows that $\tilde{\mathscr{A}}$ and $\tilde{\mathscr{B}}$ are independent over $\tilde{\mathscr{F}}$.

The converse is clear.

(2) Let $q \in \mathscr{A}, f \in \mathscr{F}$, and $h \in \mathscr{B}$. Then

$$
E_{\mathscr{F}}(g f h)=E_{\mathscr{F}}(g h) f=E_{\mathscr{F}}(g) E_{\mathscr{F}}(h) f=E_{\mathscr{F}}(g f) E_{\mathscr{F}}(h)
$$

Thus $g f$ and $h$ are independent over $\mathscr{F}$ whence so are $\sum_{i=1}^{n} g_{i} f_{i}$ and $h$ where $g_{i} \in \mathscr{A}$, $f_{i} \in \mathscr{F}, 1 \leq i \leq n$. Since $\sum g_{i} f_{i}$ is a typical element of $\mathscr{A} \vee \mathscr{F}$, this shows that $\mathscr{A} \vee \mathscr{F}$ and $\mathscr{B}$ are independent over $\mathscr{F}$. Finally another application of this argument serves to complete the proof.

(2.10) Theorem. Let $\mathscr{A}, \mathscr{B}$, and $\mathscr{F}$ be subalgebras of $\mathcal{U}$ with $\mathscr{F} \subset \mathscr{A} \cap \mathscr{B}$. Then $\mathscr{A}$ and $\mathscr{B}$ are independent over $\mathscr{F}$ if and only if $\tilde{\mathscr{A}} \cap \tilde{\mathscr{B}}=\tilde{\mathscr{F}}, \tilde{\mathscr{A}}$ and $\tilde{\mathscr{B}}$ are disjoint over $\tilde{\mathscr{F}}$, and $m \kappa^{-1}=m_{\tilde{\mathscr{A}}} \otimes \tilde{\mathscr{F}} m_{\tilde{\mathscr{B}}}$, where $\kappa: U \rightarrow|\mathscr{A}| \times|\mathscr{B}|$ is the canonical map.

Proof. Necessity. By (2.9) $\tilde{\mathscr{A}}$ and $\tilde{\mathscr{B}}$ are independent over $\tilde{\mathscr{F}}$.

Since $\tilde{\mathscr{A}}$ and $\tilde{\mathscr{B}}$ are closed, $\tilde{\mathscr{F}} \subset \tilde{\mathscr{A}} \cap \tilde{\mathscr{B}}=\mathscr{G}$. Then

$$
\kappa(U) \subset|\tilde{A}| \times_{\mathscr{G}}|\tilde{\mathscr{B}}| \subset|\tilde{\mathscr{A}}| \times_{\tilde{F}}|\tilde{\mathscr{B}}| \text {. }
$$

But $\kappa(U)=|\tilde{A}| \times \tilde{F}|\tilde{\mathscr{B}}|$ by (2.7). Now if $\tilde{\mathscr{F}}$ were not equal to $\mathscr{G}$, then there would exist distinct elements $g_{1}, g_{2}$ in $|\mathscr{G}|$ with the same image in $|\tilde{F}|$. Let $a \in|\tilde{A}|, b \in|\tilde{\mathscr{B}}|$ with $a \mathscr{G}=g_{1}, b \mathscr{G}=g_{2}$. Then $(a, b) \in|\tilde{A}| \times_{\mathscr{F}}|\tilde{\mathscr{B}}| \backslash|\tilde{A}| \times_{\mathscr{G}}|\tilde{\mathscr{B}}|$ which is a contradiction. Thus $\tilde{\mathscr{F}}=\tilde{\mathscr{A}} \cap \tilde{\mathscr{B}}$.

The rest of (2.10) now follows immediately from (2.7).

\section{Almost periodicity and discrete spectrum}

In this section we begin the study proper of the relation between certain concepts employed in topological dynamics and ergodic theory. The first of these to be discussed is almost periodicity (or equicontinuity) and the corresponding ergodic theory concept of discrete spectrum.

A flow $(X, T)$ is equicontinuous or almost periodic if the family of maps $x \rightarrow x t: X \rightarrow$ $X$ is equicontinuous. It turns out that this is so if and only if $C(X)$ is the uniform closure of the span of the (continuous) eigenfunctions on $X$. (A function $f \in C(X)$ is an eigenfunction if $\operatorname{span}\{t f \mid t \in T\}$ is finite dimensional.)

Now an ergodic 'process' $(X, T, \mu)$ has discrete spectrum if $L^{2}(X)$ is spanned in the Hilbert space sense by the $L^{2}$-eigenfunctions. $\left(f \in L^{2}(X)\right.$ is an $L^{2}$-eigenfunction if $\operatorname{span}\{t f \mid t \in T\}$ is a finite dimensional subspace of $L^{2}(X)$.)

The similarity of the two notions is of course striking but it becomes even more so if we consider the latter in terms of $C(\tilde{X})$ where $\tilde{X}$ is the Gelfand space of $L^{\infty}(X, \mu)$. Then $C(\tilde{X})=L^{\infty}(\tilde{X})$ and the two notions of eigenfunction coincide. 
What should discrete spectrum mean in terms of $C(\tilde{X})$ ? Of course it is not 'reasonable' to expect $C(\tilde{X})$ to be the uniform closure of the span of its eigenfunctions. The correct requirement is that it be the $\mathscr{T}_{m}$-closure of the latter. This means that there is a compact Hausdorff process $(Y, T, \nu)$ such that the flow $(Y, T)$ is equicontinuous and $L^{\infty}(X) \cong L^{\infty}(Y)$.

This approach leads directly to (3.10) which is a generalization of the classical result of Halmos \& Von Neumann concerning ergodic processes with discrete spectrum. (If certain countability assumptions are added, then the isomorphism above is induced by an 'isomorphism' of $X$ onto $Y$.) Moreover the proof of the ergodic theory result is deduced directly from the corresponding one in topological dynamics.

In the course of the proof it is shown (3.9) how one takes account of the topology on $T$; (see $\S 0$.)

(3.1) Standing notation. In addition to (1.1) it is henceforth assumed that a group $T$ acts on $U$ in such a way that $m$ is $T$-invariant and the flow $(U, T, m)$ is ergodic. Unless specified otherwise $T$ will assumed to be provided with the discrete topology.

Let $f \in \mathcal{U}$ and $t \in T$. Then $t f$ will denote that element of $\mathcal{U}$ such that $(t f)(x)=f(x t)$ $(x \in U)$. ( $t f \in \mathcal{U}$ since the map $(x, s) \rightarrow x s: U \times T \rightarrow U$ is assumed continuous.)

A subalgebra $\mathscr{A}$ of $\mathcal{U}$ is a $T$-subalgebra if it is invariant under $T$, i.e. $t f \in \mathscr{A}(t \in T$, $f \in \mathscr{A})$.

The invariance of $m$ implies that $m(t f)=m(f)(t \in T, f \in \mathcal{U})$.

(3.2) Remarks. (1) Let $V$ be a non-vacuous open subset of $U$. Then $\overline{V T}$ is an open-closed $T$-invariant subset of $U$ and the ergodicity of $m$ implies that its characteristic function is constant a.e. $m$. Since $m(V) \neq 0, \overline{V T}=U$. Thus the flow $(U, T)$ is topologically transitive.

(2) Let $\mathscr{A}$ be a $T$-subalgebra of $U$. Then $\overline{\mathscr{A}}$ and $\tilde{A}$ are also $T$-subalgebras of $U$. Moreover $R(\mathscr{A})$ (see (1.5)) is a closed $T$-invariant equivalence relation on $U$, whence $T$ induces an action on $|\mathscr{A}|$ such that the canonical map $U \rightarrow|\mathscr{A}|$ is a flow epimorphism, $m_{\mathscr{A}}$ is $T$-invariant and $\left(|\mathscr{A}|, T, m_{\mathscr{A}}\right)$ is ergodic. It now follows from (1) above that $(|\mathscr{A}|, T)$ is topologically transitive. Consequently if in addition $|\mathscr{A}|$ is metrizable, the flow $(|\mathscr{A}|, T)$ is point transitive; i.e. there exists $x \in|\mathscr{A}|$ with $\overline{x T}=|\mathscr{A}|$.

(3) Let $\mathscr{A}$ be a $T$-subalgebra of $U$. Then the invariance of $m$ implies that $E_{\mathscr{A}}(t f)=t E_{\mathscr{A}}(f)(t \in T, f \in \mathscr{A})$.

(4) If the algebras $\mathscr{A}, \mathscr{B}$ of theorem (2.3) are $T$-invariant, then the map $\lambda:|\tilde{A}| \rightarrow$ $M(|\tilde{\mathscr{B}}|)$ is a relatively invariant measure (RIM) i.e. $\lambda_{y t}=\lambda_{y} t$ where $\left(\lambda_{y} t\right)(f)=\lambda_{y}(t f)$, $(f \in \tilde{\mathscr{B}}, t \in T, y \in|\tilde{A}|)$.

(3.3) Definition. Let $f \in \mathcal{U}$. Then $f$ is almost periodic if $\overline{f T}$ is $\mathscr{T}_{u}$-compact.

(3.4) TheOREM. Let $\mathscr{E}$ be the set of almost periodic functions. Then:

(1) $\mathscr{E}$ is a uniformly closed T-subalgebra of $U$; and

(2) $(|\mathscr{E}|, T)$ is a compact, minimal, equicontinuous flow.

Proof. The only non-standard item of (3.4) is the minimality of $(|\mathscr{E}|, T)$. To see this set $x \equiv y(R)$ if $y \in \overline{x T}(x, y \in|\mathscr{E}|)$. Then the equicontinuity of $T$ implies that $R$ is a closed invariant equivalence relation on $|\mathscr{E}|$. If $|\mathscr{E}|$ were not minimal, $|\mathscr{E}| / R$ would 
not reduce to a point, and so would admit a non-constant continuous real valued function $f$. Then $f$ composed with the canonical map from $|\mathscr{E}|$ onto $|\mathscr{E}| / R$ would a non-constant continuous invariant function contradicting the ergodicity of $\left(|\mathscr{E}|, T, m_{\mathscr{E}}\right)$.

For the rest of (3.4) see [4] for example.

(3.5) Definition. Let $f \in \mathcal{U}$. Then $\mathscr{V}(f)$ will denote the subspace of $\mathscr{U}$ generated by $\{t f \mid t \in T\}$ and eig $(\mathscr{U})=\{f \mid \operatorname{dim} \mathscr{V}(f)<\infty\}$. (Thus eig $(\mathcal{U})$ is just the set of eigenfunctions in $U$.)

Let $\mathscr{A}$ be a $T$-subalgebra of $\mathscr{U}$. Then $\mathscr{A}$ has discrete spectrum if $\tilde{\mathscr{A}}=$ $\operatorname{cls}_{\mathscr{T}_{m}} \operatorname{span}\{\operatorname{eig}(\mathcal{U}) \cap \tilde{\mathscr{A}}\}$. (This coincides with the usual definition.)

(3.6) Remarks. Let $\mathscr{E}$ be the set of almost periodic functions. Then it follows from (3.4) that the enveloping semigroup $E=E(|\mathscr{E}|, T)$ is a compact topological group containing $T$ as a dense subgroup and that $(|\mathscr{E}|, T) \cong(E / H, T)$ for some closed subgroup $H$ of $E$. This in turn implies that $\mathscr{E}$ is the uniform closure of span $\{$ eig $(\mathcal{U})\}$.

(3.7) THEOREM. Let $\mathscr{A}$ be a T-subalgebra of $U$ with discrete spectrum and $\mathscr{B}=\tilde{\mathscr{A}} \cap \mathscr{E}$. Then $\mathscr{B}$ is a uniformly closed $T$-subalgebra of $U$ such that $\tilde{\mathscr{B}}=\tilde{\mathscr{A}}$ and $(|\mathscr{B}|, T)$ is a minimal equicontinuous flow.

Proof. Since eig $(\mathcal{U}) \subset \mathscr{E}((3.6)), \tilde{\mathscr{B}}=\tilde{\mathscr{A}}$ by (3.5). Since $\mathscr{B} \subset \mathscr{E},(|\mathscr{B}|, T)$ is a homomorphic image of $(|\mathscr{E}|, T)$ whence it is minimal and equicontinuous by (3.4).

(3.8) Definition. Let $\mathscr{V}$ be a $T$-invariant subspace of $\mathscr{U}$. Then $f \rightarrow t f: \mathscr{V} \rightarrow \mathscr{V}$ is a linear map $(t \in T)$ and thus $T$ is represented as a set of linear maps of $\mathscr{V}$ into $\mathscr{V}$. This allows one to define various natural toplogies on $T$ which all coincide when $\mathscr{V}$ is finite dimensional. In this case the topology induced on $T$ will be denoted $\tau(\mathscr{V})$.

(3.9) TheOREM. Let $\mathscr{A}$ be a T-subalgebra of $\mathcal{U}$ with $\mathscr{A}=\tilde{\mathscr{A}}$ and $\mathscr{T}$ a topology on $T$ such that $\mathscr{T} \supset \tau(\mathscr{V}(f))$ for all $f \in \mathcal{U}$ with $\operatorname{dim} \mathscr{V}(f)$ finite. Then the canonical map $\varphi:(T, \mathscr{T}) \rightarrow E(|\mathscr{A} \cap \mathscr{E}|)$ is continuous, and if moreover $\mathscr{T}$ is compact, then $\varphi$ is an epimorphism with $T / \operatorname{ker} \varphi$ isomorphic to $E(|\mathscr{A} \cap \mathscr{E}|)$.

Proof. Let $\left(t_{\alpha}\right)$ be a net in $T$ which converges to $t \in T$ with respect to $\mathscr{T}$ and let $x \in U$. Then to demonstrate the continuity of $\varphi$ it suffices to show that

$$
\lim f\left(x t_{\alpha}\right)=f(x) \quad(f \in \mathscr{A} \cap \mathscr{E}) .
$$

Let $\mathscr{B}=\{f \in \mathscr{A} \cap \mathscr{E} \mid(*)$ holds $\}$. Then $\mathscr{B}$ is clearly a uniformly closed $T$-subalgebra which by assumption contains $\{f \in \mathscr{A} \cap \mathscr{E} \mid \operatorname{dim} \mathscr{V}(f)$ finite $\}$. Since the uniform closures of the span of the latter is all of $\mathscr{A} \cap \mathscr{E}, \mathscr{B}=\mathscr{A} \cap \mathscr{E}$.

The rest of (3.9) is straightforward.

(3.10) Corollary (von Neumann, Halmos, Mackey). Let $(X, \mu)$ be a standard Borel space, $T$ a second countable locally compact topological group, $\Pi: X \times T \rightarrow X a$ Borel map which defines an ergodic action of $T$ on $X$ with discrete spectrum. Then there exists a compact topological group $G$, a closed subgroup $H$ of $G$, a representation of $T$ as a dense subgroup of $G$ and a Borel isomorphism of $(G / H, T, \lambda)$ onto $(X, T, \mu)$ where $\lambda$ is Haar measure on $G$. 
Proof. If we take $U=L^{\infty}(X, \mu)=\mathscr{A}$, then the assumptions on $\Pi$ imply that the topology on $T$ satisfies the condition of (3.9). Consequently $L^{2}(X, T, \mu) \cong$ $L^{2}(G / H, T, \lambda)$ where $G=E(|\mathscr{E}|)$ and $H$ is a closed subgroup of $G$. Theorem (3.10) now follows from Mackey's point realization theorem [9].

\section{Bitransformation groups and cocycles}

Before studying the notion of generalized discrete spectrum in this general context it is convenient to extent the results of Mackey and Zimmer on minimal cocycles [10].

The basic result is (4.6) which is the relativized version of the theorem that a measurable eigenfunction is continuous.

(4.1) Definition. A bitransformation group is a triple $(G, X, T)$ where $(G, X)$ and $(X, T)$ are two flows with the same phase space $X$ and phase groups $G$ and $T$ respectively such that $(g x) t=g(x t),(x \in X, g \in G, t \in T)$. Notice that in this case the product $G \times T$ acts on $X$ via the map $(x, g, t) \rightarrow g^{-1} x t: X \times G \times T \rightarrow X$.

(4.2) Standing assumptions. In addition to the assumptions made in $\S 3$ the following will be in force throughout this section.

(1) $(G, X, T)$ will denote a bitransformation group such that $G$ and $X$ are compact Hausdorff and $G$ acts freely on $X$.

(2) The quotient transformation group $(X / G, T)$ will be denoted $(Y, T)$ and it is assumed that $Y$ is of the form $|\mathscr{F}|$ for some $T$-subalgebra $\mathscr{F}$ of $U$ with $\mathscr{F}=\mathscr{F}$.

(3) $\mu$ will denote the Haar lift of $m$ to $X$ and $\tilde{X}$ the Gelfand space of $L^{\infty}(X, \mu)$.

(4) The canonical map of $X$ onto $Y=X / G$ will be denoted by $\Pi$.

(4.3) Remarks. (1) Let $f \in C(X), \lambda$ the Haar measure on $G$, and $\bar{f}(x)=$ $\int_{G} f(g x) d \lambda(g),(x \in X)$. Since $\vec{f}(g x)=\bar{f}(x)(g \in G, x \in X)$, it induces an element $f_{Y}$ of $C(Y)$. Then $\mu(f)$ is defined to be $m\left(f_{Y}\right)$.

The measure $\mu$ is invariant under both $G$ and $T$. It is ergodic with respect to the action of $G \times T$, but need not be with respect to the action of $T$.

(4.4) Definition. A process $(Z, T, \nu)$ is a flow $(Z, T)$ together with a $T$-invariant Borel probability measure $\nu$. A homomorphism of the process $(Z, T, \nu)$ into the process $(W, T, \gamma)$ is a homomorphism $h$ of the flow $(Z, T)$ into the flow $(W, T)$ such that $\gamma(A)=\nu\left(h^{-1}(A)\right)$ for all Borel subsets $A$ of $W$.

(4.5) LemMA. Let $(Z, T, \nu)$ be a process with compact Hausdorff phase space $Z$ and supported measure $\nu$, and $h$ a homomorphism of $(Z, T, \nu)$ onto $(Y, T, m)$ such that the induced map $h^{*}: L^{\infty}(Y) \rightarrow L^{\infty}(Z)$ is also onto. Then $h$ is an isomorphism.

Proof. Let $f \in C(Z)$. Since every $L^{\infty}$-function on $Y$ is equal almost everywhere to a continuous one, the assumption that $h^{*}$ is onto implies that there exists $g \in C(Y)$ with $g \circ h=f$ (a.e. $\nu$ ). Since $\nu$ is supported and both $f$ and $g \circ h$ are continuous, $g \circ h=f$. Thus the map of $C(Y)$ into $C(Z)$ induced by $h$ is onto whence $h$ is one-one. The proof is completed.

(4.6) TheOREM. Let $f \in C(\tilde{X})$ be such that $\operatorname{dim} \operatorname{span}\{f \alpha \mid \alpha \in G\}<\infty$. Then $f \in C(X)$. Remarks. (1) Here $(f \alpha)(x)=f(\alpha x)(\alpha \in G, x \in \tilde{X})$ and $C(X)$ is identified with a subalgebra of $C(\tilde{X})$ via the canonical map of $\tilde{X}$ onto $X$. 
(2) If we take $T=e$ and $X=G$, then we get the classical result that a measurable eigenfunction is continuous.

(3) Notice that $\operatorname{dim} \operatorname{span}\{t f \alpha \mid \alpha \in G\}<\infty(t \in T)$.

The assumption of (4.6) is carried over to the following lemmas upon which its proof is based.

(4.6.1) LeмmA. Let $\mathscr{A}$ be the uniformly closed T-subalgebra of $C(\tilde{X})$ generated by $\{f \alpha \mid \alpha \in G\} \cup C(X)$. Then $\mathscr{A}$ is invariant under $\mathrm{G} \times \mathrm{T}$ and the map $(\alpha, x) \rightarrow \varphi$ $\alpha x: G \times|\mathscr{A}| \rightarrow|\mathscr{A}|$ is continuous.

Proof. By definition $\mathscr{A}$ is invariant under $T$. Its invariance under $G \times T$ now follows from the invariance of $\{f \alpha \mid \alpha \in G\} \cup C(X)$ under $G$.

To prove $\varphi$ continuous it suffices to show that it is unilaterally continuous, [3]. Let $\left(\alpha_{n}\right)$ be a net in $G$ which converges to $\alpha \in G, x \in|\mathscr{A}|$, and $\mathscr{B}=$ $\left\{h \in \mathscr{A} \mid h\left(\alpha_{n} x\right) \rightarrow h(\alpha x)\right\}$. Then it is not hard to see that $\mathscr{B}$ is a uniformly closed $T$-invariant algebra containing $\{f \alpha \mid \alpha \in G\} \cup C(X)$. Hence $\mathscr{B}=\mathscr{A}$ and so the map $\alpha \rightarrow \alpha x: G \rightarrow|\mathscr{A}|$ is continuous $(x \in|\mathscr{A}|)$. Since $x \rightarrow \alpha x:|\mathscr{A}| \rightarrow|\mathscr{A}|$ is continuous $(\alpha \in G)$, the proof is completed.

The proof of the next lemma is standard and so will be omitted.

(4.6.2) LeMMA. Let $(G, W, \nu)$ be a process with compact acting group $G$, compact Hausdorff space $W$ and Borel probability measure $\nu$. Then the canonical map $\Pi: W \rightarrow$ $W / G$ induces a monomorphism $\Pi^{*}: L^{2}(W / G, \eta) \rightarrow L^{2}(W, \nu)$ with

$$
\text { im } \left.\Pi^{*}=\{h \mid h=h \alpha \quad \text { (a.e. } \nu)(\alpha \in G)\right\} \text {. }
$$

( Here $\eta=\nu \Pi^{-1}$.)

Proof of (4.6). Let $\mathscr{A}$ be as in lemma (4.6.1). Then the diagram:

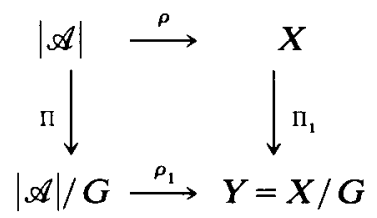

is commutative where $\Pi, \rho, \Pi_{1}$ are the canonical maps and $\rho_{1}$ is induced by $\rho$. Passing to $L^{2}$ yields the commutative diagram:

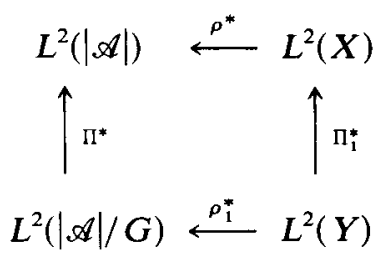

Now let $f \in L^{2}(|\mathscr{A}| / G)$. Then $\Pi^{*}(f)$ is $G$-invariant. Since $\mathscr{A} \subset C(\tilde{X}), \rho^{*}$ is an isomorphism onto. Hence there exists a $G$-invariant element $h$ of $L^{2}(X)$ with $\rho^{*}(h)=\Pi^{*}(f)$. By lemma (4.6.2) there exists $g \in L^{2}(Y)$ with $\Pi_{1}^{*}(g)=h$. Then $\Pi^{*} \rho_{1}^{*}(g)=\rho^{*} \Pi_{1}^{*}(g)=\rho^{*} h=\Pi^{*} f$, whence by lemma (4.6.2) $\rho_{1}^{*}(g)=f$. Thus $\rho_{1}^{*}$ is onto and so by (4.5) $\rho_{1}$ is an isomorphism.

Now let $z_{1}, z_{2} \in|\mathscr{A}|$ with $\rho\left(z_{1}\right)=\rho\left(z_{2}\right)$. Then $\rho_{1}\left(\Pi z_{1}\right)=\Pi_{1} \rho\left(z_{1}\right)=\Pi_{1} \rho\left(z_{2}\right)=\rho_{1}\left(\Pi z_{2}\right)$ whence $\Pi z_{1}=\Pi z_{2}$. This implies that $z_{2}=\alpha z_{1}$, for some $\alpha \in G$. Consequently 
$\alpha \rho\left(z_{1}\right)=\rho\left(\alpha z_{1}\right)=\rho\left(z_{2}\right)=\rho\left(z_{2}\right)=\rho\left(z_{1}\right)$ whence $\alpha=e$ since $G$ acts freely on $X$. Thus $z_{1}=z_{2}$ which means that $\rho$ is one-one; i.e. $\mathscr{A}=C(X)$. The proof is completed.

(4.7) THEOREM. Let $f$ be a T-invariant element of $C(\tilde{X})$ with $\operatorname{dim} \operatorname{span}\{f \alpha \mid \alpha \in G\}$ finite, $\mathscr{D}$ the $T$-subalgebra of $C(X)$ generated by $\{f \alpha \mid \alpha \in G\}, \psi: X \rightarrow|\mathscr{D}|$ the canonical map, $d \in|\mathscr{D}|, Z=\varphi^{-1}(d)$, and $H=\{\alpha \in G \mid \alpha d=d\}$. Then:

(1) $\mathscr{D}$ is a $G \times T$-invariant subalgebra of $C(X)$ and $\varphi$ is a $G \times T$-epimorphism.

(2) $G d=|\mathscr{D}|$ i.e. $G$ acts transitively on $|\mathscr{D}|$.

(3) $\alpha Z \cap Z \neq 0$ if and only if $\alpha \in H$.

(4) $Z$ is closed and T-invariant.

(5) $(H, Z, T)$ is a bitransformation group.

(6) $\Pi\left(z_{1}\right)=\Pi\left(z_{2}\right)$ if and only if $z_{2} \in H z_{1}\left(z_{1}, z_{2} \in Z\right)$ where $\Pi: X \rightarrow Y$ is the canonical map.

Proof. (1) This follows from the definition of $\mathscr{D}$.

(2) Since $t f \alpha=f \alpha(t \in T, \alpha \in G)$, all the elements of $\mathscr{D}$ are $T$-invariant. This implies that $T$ acts trivially on $|\mathscr{D}|$. Hence $(G,|\mathscr{D}|)$ is minimal and (2) follows from the compactness of $G$.

(3) Let $\alpha \in G, z \in \alpha Z \cap Z$. Then $z=\alpha u$ for some $u \in Z$ and $\alpha d=\alpha \varphi(u)=\varphi(\alpha u)=$ $\varphi(z)=d$, whence $\alpha \in H$. On the other hand if $\alpha \in H$ then $\alpha z \in Z(z \in Z)$.

(4) and (5) are evident.

(6) Let $z_{1}, z_{2} \in Z$. Then $\Pi\left(z_{1}\right)=\Pi\left(z_{2}\right)$ if and only if $z_{2}=\alpha z_{1}$, for some $\alpha \in G$. But then $\alpha \in H$ by (3).

(4.8) Definition. Let $(W, T)$ be a flow and $G$ a topological group. A cocycle on $W$ to $G$ is a continuous function $\sigma: W \times T \rightarrow G$ such that

$(x \in W, t, s \in T)$.

$$
\sigma(x, t s)=\sigma(x, t) \sigma(x t, s) \text { and } \sigma(x, e)=e,
$$

Two cocycles $\sigma, \eta$ on $W$ to $G$ are cohomologous if there exists a homeomorphism $\varphi: W \rightarrow W$ such that

$$
\varphi(w) \sigma(w, t)=\eta(w, t) \varphi(w t) \quad(w \in W, t \in T) .
$$

Let $\sigma$ be a cocycle on $(W, T)$ to $G$. Then the maps $(\beta, \alpha, w) \rightarrow(\beta \alpha, w): G \times G \times W \rightarrow$ $G \times W$ and $(\alpha, w, t) \rightarrow(\alpha \sigma(w, t), w t): G \times W \times T \rightarrow G \times W$ define a bitransformation group structure on $G \times W$ which is denoted $\left(G, G \times{ }_{\sigma} W, T\right)$. The projection $G \times W \rightarrow$ $W$ induces an isomorphism of $\left(G \times_{\sigma} W / G, T\right)$ onto $(W, T)$.

(4.9) Theorem. There exists a cocycle $\sigma$ on $Y$ to $G$ such that $(G, X, T)$ is isomorphic to $\left(G, G \times{ }_{\sigma} Y, T\right)$ via an isomorphism which induces the identity on $Y$. Any two such cocycles are cohomologous.

Proof. Let $\Pi: X \rightarrow Y$ be the canonical map. Then there exists a continuous map $\delta: Y \rightarrow X$ with $y=\Pi(\delta(y))(y \in Y)$ [6]. Define $\sigma$ by the equations:

and

$$
\sigma(y, t) \delta(y, t)=\delta(y) t \quad(y \in Y, t \in T)
$$

$$
\varphi(\alpha, y)=\alpha \delta(y) \quad(\alpha \in G, y \in Y) .
$$

Then it is standard that $\varphi$ is a homeomorphism of $G \times Y$ onto $X$. Also

$$
\varphi(\beta(\alpha, y))=\varphi(\beta \alpha, y)=\alpha \delta(y)=\beta \varphi(\alpha, y),
$$


and

$$
\varphi((\alpha, y) t)=\varphi(\alpha \sigma(y, t), y t)=\alpha \sigma(y, t) \delta(y t)=\alpha \delta(y) t=\varphi(\alpha, y) t
$$

( $\alpha, \beta \in G, y \in Y, t \in T$ ) shows that $\varphi$ is a bitransformation group isomorphism of $\left(G \times{ }_{\sigma} Y, T\right)$ onto $(X, T)$.

Finally $\Pi \varphi(\alpha, y)=\Pi(\alpha \delta(y)) \Pi \delta(y)=y(\alpha \in G, y \in Y)$ implies that $\varphi$ induces the identity on $Y$.

Now let $\gamma$ be a cocycle on $Y$ to $G$ and $\xi$ an isomorphism of $\left(G, G \times{ }_{\gamma} Y, T\right)$ onto $(X, T)$ such that $\Pi(\xi(\alpha, y))=y(\alpha \in G, y \in Y)$. Set $\varphi(y)=\xi(e, y)$. Then $\Pi \varphi(y)=y$ and $\gamma(y, t) \varphi(y t)=\varphi(y) t(y \in Y, t \in T)$. Since $\Pi \varphi(y)=y=\Pi \delta(y)(y \in Y)$, there exists a function $f: Y \rightarrow G$ with $\varphi(y)=f(y) \delta(y)(y \in Y)$. Since $G$ acts freely on $X$ and $\varphi, \delta$ are continuous, so is $f$. Then

$$
\gamma(y, t)^{-1} \varphi(y) t=\varphi(y t)=f(y t) \delta(y t)=f(y t) \sigma(y, t)^{-1} \delta(y) t
$$

whence

$$
\gamma(y, t)^{-1} f(y)=f(y t) \sigma(y, t)^{-1} \quad(y \in Y, t \in T)
$$

so that $\sigma$ and $\delta$ are cohomologous. The proof is complete.

(4.10) Definition. Let $H$ be a closed subgroup of $G$. Then a reduction of $G$ to $H$ is a subflow $(H, Z, T)$ of $(G, X, T)$ such that $Z$ is a closed subset of $X$ and the canonical map $I I$ induces an isomorphism of $(Z / H, T)$ onto $(Y, T)$. The flow $(G, X, T)$ is irreducible if $G$ cannot be reduced to any proper subgroup.

Notice that in this case, $\alpha Z \cap Z \neq \varnothing$ if and only if $\alpha \in H$.

(4.11) Lemma. Let $\nu$ be a T-invariant probability measure on $X$ with $\nu \Pi^{-1}=m$. Then $\nu=\mu$ if and only if $\alpha \nu=\nu(\alpha \in G)$.

Proof. Necessity is clear. To prove sufficiency let $f \in C(X)$. Set $F(\alpha, x)=f(\alpha x)$ $(\alpha \in G, x \in X)$. Then $F \in C(G \times X)$ and so by Fubini's theorem

$$
I=\int_{G} \int_{X} F(\alpha, x) d \nu(x) d \lambda(\alpha)=\int_{X} \int_{G} F(\alpha, x) d \lambda(\alpha) d \nu(x)=J,
$$

where $\lambda$ is Haar measure on $G$.

Since $\alpha \nu=\nu$,

$$
\int_{X} F(\alpha, x) d \nu(x)=\int_{X} f(\alpha x) d \nu(x)=\int_{X} f(x) d \nu(x) \quad(\alpha \in G)
$$

whence $I=\int_{X} f(x) d \nu(x)$. The map

$$
x \rightarrow \int_{G} F(\alpha, x) d \lambda(\alpha)=\int_{G} f(\alpha x) d \lambda(\alpha)
$$

is continuous and invariant under $G$ whence

$$
\begin{aligned}
J & =\int_{Y} \int_{G} f(\alpha x) d \lambda(\alpha) d \nu \Pi^{-1}(x)=\int_{Y} \int_{G} f(\alpha x) d \lambda(\alpha) d m(x) \\
& =\int_{X} f(x) d \mu(x) .
\end{aligned}
$$

The proof is complete. 
(4.12) THEOREM. The following are pairwise equivalent:

(1) $(G, X, T)$ is irreducible.

(2) $\mu$ is T-ergodic.

(3) $M(X)=\{\mu\}$ where $M(X)$ is the set of T-invariant probability measures $\nu$ on $X$ with $\nu \Pi^{-1}=m$.

Proof. (1) implies (2). If $\mu$ were not ergodic the $G$ invariant subspace $\mathscr{H}=$ $\left\{f \in L_{2}(X) \mid t f=f\right.$ a.e. $\left.(t \in T)\right\}$ of $L_{2}(X)$ would contain non-constant functions. Since $G$ is compact there would be a non-constant $f$ in $\mathscr{H}$ with $\operatorname{dim} \operatorname{span}\{f \alpha \mid \alpha \in G\}<\infty$. By (4.7) there would then be a non-trivial reduction of $G$ thus contradicting (1).

(2) implies (3). Let $\nu \in M(X)$. Then $\int_{G}(\alpha \nu) d \lambda(\alpha)$ is a $G$-invariant element of $M(X)$. Hence $\mu=\int_{G}(\alpha \nu) d \lambda(\alpha)$ by (4.11). Since (2) implies that $\mu$ is an extreme point of $M(X)$ and $\mu \in \overline{\mathrm{cnv}}(\mathrm{G} v), \mu \in G \nu$. Consequently $\mu=\nu$.

(3) implies (1). Let $(H, Z, T)$ be a reduction of $(G, X, T)$ with $H \neq G$. Let $\omega$ be the Haar lift of $m$ to $Z$ and set $\nu(A)=\omega(A \cap Z)$ for all Borel subsets $A$ of $X$. Then $\nu \in M(X)$ with $\nu \neq \mu$ which contradicts (3).

(4.13) TheOREM. There exists an irreducible subflow $(H, Z, T)$ of $(G, X, T)$ such that:

(1) $\{\alpha Z \mid \alpha \in G\}$ is a partition of $X$; and

(2) if $(E, W, T)$ is an irreducible subflow of $(G, X, T)$, then $W=\alpha Z$ and $K=$ $\alpha H \alpha^{-1}$ for some $\alpha \in G$.

Proof. Let $\mathscr{E}$ be the collection of subflows $(L, N, T)$ of $(G, X, T)$ such that $(L, N, T)$ is a reduction of $G$ to $L$. For $\left(L_{1}, N_{1}, T\right),\left(L_{2}, N_{2}, T\right) \in \mathscr{E}$ set $\left(L_{1}, N_{1}, T\right) \leq$ $\left(L_{2}, N_{2}, T\right)$ if $L_{1} \subset L_{2}$ and $N_{1} \subset N_{2}$. Then it is straightforward that $(\mathscr{E}, \leq)$ is inductive, whence by Zorn's lemma there exists a minimal element $(H, Z, T)$ in $\mathscr{E}$. Then $(H, Z, T)$ is clearly irreducible and (1) follows from the fact that $\alpha Z \cap Z \neq \varnothing$ if and only if $\alpha \in H$.

(2) Let $(E, W, T)$ be an irreducible reduction of $(G, X, T)$. Then by (4.12) the Haar lifts $\omega, \nu$ of $m$ to $Z$ and $W$ respectively are $T$-ergodic probability measures on those spaces. Then $\omega, \nu$ may be viewed as elements of $M(X)$ and as such remain $T$-ergodic.

Now $\mu=\int_{G}(\alpha \omega) d \lambda(\alpha)=\int_{G}(\alpha \nu) d \lambda(\alpha)$ implies that $G \nu=G \omega$ since $M(X)$ is a simplex [8]. Consequently there exists $\alpha \in G$ with $\alpha \nu=\omega$. Then

$$
1=\nu(Z)=\left(\alpha^{-1} \omega\right)(Z)=\omega(\alpha Z)
$$

whence $\omega(\alpha Z \cap W)=1$. Since $W \backslash \alpha Z \cap W$ is an open $T$-invariant subset of $W$ with $\omega(W \backslash \alpha Z \cap W)=0$ and $\omega$ is supported, $W \backslash \alpha Z \cap W=\varnothing$ and so $W \subset \alpha Z$. A similar argument shows that $\alpha Z \subset W$; whence $W=\alpha Z$.

Finally $\beta \in K$ if and only if $\beta W \cap W \neq \varnothing$ and $\gamma \in H$ if and only if $\gamma Z \cap Z \neq \varnothing$ together with $W=\alpha Z$ imply that $K=\alpha H \alpha^{-1}$. The proof is completed.

(4.14) Definition. Let $\sigma$ be a cocycle on $Y$ to $G$. Then $G(\sigma)$ will denote the set of closed subgroups $\left\{\alpha H \alpha^{-1} \mid \alpha \in G\right\}$ such that $(H, Z, T)$ is an irreducible reduction of $\left(G, G \times_{\sigma} Y, T\right)$. The cocycle $\sigma$ is minimal if there exists $K \in G(\sigma)$ such that $\sigma(y, t) \in K(y \in Y, t \in T)$. 
(4.15) THEOREM. Let $\sigma$ be a cocycle on $Y$ to $G$. Then there exists a minimal cocycle $\delta$ on $Y$ to $G$ with $\delta$ and $\sigma$ cohomologous.

Proof. By (4.13) there exists an irreducible subflow $(H, Z, T)$ of the flow $\left(G, G \times{ }_{\sigma} Y, T\right)$. As in (4.9) there exists a continuous section $\rho: Y \rightarrow Z \subset X$. Then the induced cocycle $\delta: Y \times T \rightarrow G$ defined by the equation

$$
\delta(y, t) \rho(y t)=\rho(y) t \quad(y \in Y, t \in T)
$$

takes its values in $H$ and $\rho$ and $\sigma$ are cohomologous by (4.9).

(4.16) TheOrem. Let $(E, T)$ be a flow, $\psi:(X, T) \rightarrow(E, T) \varphi:(E, T) \rightarrow(Y, T)$ epimorphisms with $\Pi=\varphi \circ \psi ;(W, T)$ a subflow of $(E, T), \nu$ a supported, ergodic, $T$-invariant measure on $W$ with $m=\nu \varphi^{-1}$ and suppose that the set $M$ of $T$-invariant probability measures $\gamma$ on $X$ with $\gamma \varphi^{-1}=\nu$ is non-empty. Then there exists an irreducible subflow $(\mathrm{H}, \mathrm{Z}, \mathrm{T})$ of $(G, X, T)$ such that $\varphi(Z)=W$ and $\nu=\omega \varphi^{-1}$ where $\omega$ is the Haar lift of $m$ to $Z$.

Proof. Since $M$ is compact and convex, there exists an extreme point $\omega$ of $M$. It is immediate that $\omega$ is an extreme point in the set of probability measures on $X$. Hence $\omega$ is ergodic.

Let $(K, N, T)$ be an irreducible subflow of $(G, X, T)$ and $\rho$ the Haar lift of $m$ to $N$. Then $\rho$ and $\omega$ are both $T$-ergodic lifts of $m$ to $X$ whence $\omega=\alpha \rho$ for some $\alpha \in G$ (see proof of (2) of (4.13)). Consequently $\omega$ is the Haar lift of $m$ to the irreducible flow $(H, Z, T)$ where $Z=\alpha(N)$ and $H=\alpha K \alpha^{-1}$.

Now $\psi(Z)$ is a closed $T$-invariant subset of $Y$ such that

$$
\nu(\psi(Z))=\omega \psi^{-1}(\psi(Z))=1
$$

Hence $W \subset \psi(Z)$ since $W=\operatorname{supp} \nu$.

On the other hand $\psi^{-1}(W)$ is a closed $T$-invariant subset of $X$ with

$$
\omega\left(\psi^{-1}(W)\right)=\nu(W)=1 .
$$

Hence $Z \subset \psi^{-1}(W)$.

Thus $W=\psi(Z)$ and the proof is complete.

\section{Relatively discrete spectrum}

In this section the results of $\S 3$ are 'relativized'. The idea of relativizing notions arose in topological dynamics as a result of attempts to generalize Furstenberg's structure theorem for minimal distal flows. In place of almost periodic flows one was lead to consider almost periodic extensions $(X, T)$ of a given flow, $(Y, T)$. The latter are so defined that they reduce to the former when $Y$ is the trivial one point flow.

The fundamental result is: let $(X, T)$ be a minimal almost periodic extension of $(Y, T)$ then there exists a bitransformation group $(G, Z, T)$ and a closed subgroup $H$ of $G$ such that $G$ is compact, $(Z, T)$ minimal, $(Z / G, T) \cong(Y, T)$ and $(Z / H, T) \cong$ $(X, T)$.

This section addresses the problem of translating this result into the ergodic theory framework. To this end it is first necessary to come up with the ergodic theoretic counterpart of an almost periodic extension. Zimmer [10] does this in the context 
of standard Borel spaces with the notion of relatively discrete spectrum and he proves a result analogous to the fundamental one quoted above.

If one examines his definition one sees that it is equivalent to the following: let $\varphi:(X, T, \mu) \rightarrow(Y, T, \nu)$ be an epimorphism of ergodic flows with $\nu=\mu \varphi^{-1}$, then $(X, T, \mu)$ has relatively discrete spectrum over $(Y, T, \nu)$ if there exist a family $\left(\mathscr{M}_{i}\right)$ of subspaces of $L^{2}(X)$ such that $L^{2}(X)$ is the Hilbert space sum of the $\mathscr{M}_{i}$ and each $\mathcal{M}_{i}$ is $T$-invariant and finitely generated as a module over $\varphi^{*}\left(L^{\infty}(Y)\right)$.

Under these conditions Zimmer [10, theorem 4.3] proves that there exists a compact group $K$, a closed subgroup $H$ of $K$ and a minimal cocycle $\alpha: Y \times T \rightarrow K$ such that $X$ is essentially isomorphic as an extension of $Y$ to $K / H \times{ }_{\alpha} Y$.

In our context the assumptions on the family $\left(\mathscr{M}_{i}\right)$ amount to requiring that its union generate $L^{\infty}(X)$ as a $\mathscr{T}_{m}$-closed, $T$-invariant conjugated closed algebra. The principal result of this section $((5.36)$ below) is a structure theorem analogous to Zimmer's with $X$ and $Y$ replaced by compact Hausdorff spaces $(\tilde{X}, \tilde{\mu})(\tilde{Y}, \tilde{\nu})$ respectively such that $L^{\infty}(\tilde{X}) \cong L^{\infty}(X), L^{\infty}(\tilde{Y}) \cong L^{\infty}(Y)$.

(5.1) Notation. Throughout this section the following notation will be in force: $\mathscr{F}$ a fixed $T$-invariant subalgebra of $U$ with $\mathscr{F}=\tilde{F}$ and $|\mathscr{F}|=Y, \Pi: U \rightarrow Y$ the canonical map, $f_{y}$ the restriction of $f$ to $\Pi^{-1}(y)(f \in \mathcal{U}, y \in Y), U_{y}=\left\{f_{y} \mid f \in \mathscr{U}\right\}(y \in Y), \underline{U}$ the disjoint union $+\left\{\mathcal{u}_{y} \mid y \in Y\right\}, \kappa: \underline{u} \rightarrow Y$, the canonical map.

The sup norm on $\mathcal{U}$ induces a norm, \|\|$_{y}$ on $U_{y}$ which makes it a Banach space $(y \in Y)$. A topology $\mathscr{T}$ is defined on $\underline{U}$ by specifying that $W \in \mathscr{T}$ if given $\alpha \in W$ there exists $f \in \underline{U}$, and $\varepsilon>0$ with $\alpha \in(f, \varepsilon)=\left\{g_{y} \mid\|f-g\|<\varepsilon\right\} \subset W$.

The following remarks are standard (see e.g. [7]) and so the proofs will be omitted. (Recall that the map $\Pi$ is open.)

(5.2) Remarks. (1) $\mathscr{T}$ is a topology on $\underline{U}$.

(2) The inclusion map $\left(\mathcal{U}_{y},\|,\|_{y}\right) \rightarrow(\mathcal{U}, \mathscr{T})$ is a homeomorphism into.

(3) The map $(\alpha, \beta) \rightarrow \alpha+\beta: \underline{\mathcal{U}} \times_{Y} \underline{\mathcal{U}} \rightarrow \underline{\mathcal{U}}$ is continuous, where $\underline{\mathcal{U}} \times_{Y} \underline{\mathcal{U}}=$ $\{(\alpha, \beta) \mid \kappa(\alpha)=\kappa(\beta)\} \subset \underline{u} \times \underline{\underline{u}}$.

(4) The maps $(c, \alpha) \rightarrow c \alpha: \mathbb{C} \times \underline{U} \rightarrow \underline{U}$ and $\alpha \rightarrow\|\alpha\|: \underline{U} \rightarrow \mathbb{R}$ are continuous.

(5) The canonical map $\kappa: \underline{U} \rightarrow Y$ is both continuous and open.

(5.3) Definition. Let $\mathscr{A} \subset \mathscr{U}$. Then $\mathscr{A}_{y}=\left\{f_{y} \mid f \in \mathscr{A}\right\} \subset \mathcal{U}_{y}$ and $\mathscr{A}=+\mathscr{A}_{y} \subset \underline{u}$. Let $\varnothing \neq$ $N \subset Y$. Then $\Gamma(N, \mathscr{A})=\{\sigma \mid \sigma: N \rightarrow \mathscr{A}$ is continuous and $\kappa \sigma(y)=y(y \in N)\}$. If $f \in \mathcal{U}$ then $\sigma_{f}$ will denote the map $y \rightarrow f_{y}: Y \rightarrow \underline{U}(f \in \mathcal{U})$. Notice that $\sigma_{f} \in \Gamma(Y, \mathcal{U})$.

(5.4) THEOREM. Let $N$ be a non-vacuous subset of $Y$ and $\sigma \in \Gamma(N, \mathcal{U})$. Then the map $x \rightarrow{ }_{F} \sigma(\Pi x)(x): \Pi^{-1}(N) \rightarrow \mathbb{C}$ is continuous.

Proof. Let $y=\Pi(x) \in N$ and $\varepsilon>0$. Let $f \in \mathcal{U}$ with $\sigma(y)=f_{y}$. Choose $K$ a neighbourhood of $y$ with $\sigma(K \cap N) \subset(f, \varepsilon / 2)$ and $W$ a neighbourhood of $x$ with $\Pi(W) \subset K$ and $|f(w)-f(x)|<\varepsilon / 2(w \in W)$. Let $w \in W \cap \Pi^{-1}(N), u=\Pi(w) \in N$, and $\sigma(u)=g_{u}$ for some $g \in \mathcal{U}$. Then $\|g-f\|<\varepsilon / 2$ implies that

$$
\begin{aligned}
|F(w)-F(x)| & =|\sigma(\Pi w)(w)-\sigma(\Pi x)(x)| \\
& =\left|g_{u}(w)-f_{y}(x)\right|=|g(w)-f(x)| \\
& \leq|g(w)-f(w)|+|f(w)-f(x)| \\
& <\varepsilon / 2+\varepsilon / 2=\varepsilon .
\end{aligned}
$$


(5.5) Corollary. Let $N$ be a non-vacuous closed subset of $Y, \mathscr{A}$ a uniformly closed, conjugate closed subalgebra of $U$ containing $\mathscr{F}$, and $\sigma \in \Gamma(N, \mathscr{A})$. Then there exists $f \in \mathscr{A}$ with $\sigma=\sigma_{f}$.

Proof. Let $F(x)=\sigma(\Pi x)(x)\left(x \in \Pi^{-1}(N)\right)$. Then by (5.4) $F$ is continuous.

Now let $u, v \in \Pi^{-1}(N)$ with $f(u)=f(v)(f \in \mathscr{A})$. Then $\Pi(u)=\Pi(v)=y$ for some $y \in Y$ since $\mathscr{F} \subset \mathscr{A}$. Hence

$$
F(u)=g_{y}(u)=g(u)=g(v)=g_{y}(v)
$$

where $g \in \mathscr{A}$ with $\sigma(y)=g_{y}$. Consequently $F=f \mid N$ for some $f \in \mathscr{A}$ by the StoneWeierstrass theorem. Thus

$$
\sigma_{f}(y)(x)=f_{y}(x)=f(x)=F(x)=\sigma(\Pi x)(x)=\sigma(y)(x)
$$

$\left(y \in N, x \in \Pi^{-1}(y)\right)$. The proof is complete.

(5.6) Definition. The action of $T$ on $\underline{\underline{U}}$. It is immediate that

$$
(\alpha, t) \rightarrow \alpha t=\left(t^{-1} f\right)_{y t}: \underline{U} \times T \rightarrow \underline{U}
$$

is a well defined action of $T$ on $\underline{U}$ where $y=\kappa(\alpha)$ and $f \in \mathcal{U}$ with $\alpha=f_{y}$. Moreover the map $\alpha \rightarrow \alpha t: \mathscr{U}_{y} \rightarrow \mathscr{U}_{y t}$ is an isometry $(y \in Y, t \in T)$.

Definition. For $f, g \in \mathcal{U}$ and $y \in Y$ set

$$
(f \mid g)_{y}=E_{\mathscr{F}}(f \bar{g})(y)=\int_{U} f \bar{g} d \lambda_{y}=\int_{U} f_{y} \bar{g}_{y} d \lambda_{y}
$$

Here $\lambda_{y}$ is the measure on $U$ induced by the linear functional $h \rightarrow\left(E_{\mathscr{F}} h\right)(y): \mathcal{U} \rightarrow \mathbb{C}$. (The last equality follows from the fact that $\operatorname{supp} \lambda_{y} \subset \Pi^{-1}(y)(y \in Y)$.)

The following remarks follow from the definitions and the results of $\S 2$.

(5.8) Remarks. (1) The map $\left(f_{y}, g_{y}\right) \rightarrow(f \mid g)_{y}: U_{y} \times U_{y} \rightarrow \mathbb{C}$ defines an inner product $(y \in Y)$.

(2) $(\alpha \mid \beta)_{y}=(\alpha t \mid \beta t)_{y}\left(\alpha, \beta \in \mathcal{U}_{y}, t \in T\right)$.

(3) The map $y \rightarrow(f \mid g)_{y}: Y \rightarrow \mathbb{C}$ is continuous $(f, g \in \mathcal{U})$.

(5.9) Standing assumption. Throughout the rest of this section $\mathcal{M}$ will denote a subset of $U$ such that: (i) $\mathscr{F} \subset \mathcal{M}$, (ii) $g+h \in \mathcal{M}$ ( $g, h \in \mathcal{M})$, (iii) $f g \in \mathcal{M}(f \in \mathscr{F}, g \in \mathcal{M}$ ) (iv) there exist $g^{1}, \ldots, g^{k} \in \mathcal{M}$ such that $\mathscr{F} g^{1}+\cdots+\mathscr{F} g^{k}=\mathcal{M}$, (v) $t \mathcal{M}=\mathcal{M}(t \in T)$. Statements (i) through (iv) say that $\mathcal{M}$ is a finitely generated $\mathscr{F}$-module and (v) that it is $T$-invariant. Such a set will be called a finitely generated $T$-F module.

(5.10) Theorem. The set $\mathscr{M}_{y}=\left\{g_{y} \mid g \in \mathcal{M}\right\}$ is a subspace of $\mathscr{U}_{y}$ with $\operatorname{dim} \mathscr{M}_{y} \leq k$ $(y \in Y)$.

Proof. That $\mathcal{M}_{y}$ is a subspace of $\mathcal{U}_{y}(y \in Y)$ follows immediately from (i) and (iii) of (5.9).

Now let $g \in \mathcal{M}$. Then by (iv) of (5.9) $g=f^{1} g^{1}+\cdots+f^{k} g^{k}$ for some $f^{1}, \ldots, f^{k} \in \mathscr{F}$. Hence $g_{y}=f_{y}^{1} g_{y}^{1}+\cdots+f_{y}^{k} g_{y}^{k} \in \mathbb{C} g_{y}^{1}+\cdots+\mathscr{C} g_{y}^{k}(y \in Y)$.

Henceforth $r$ will denote $\max \left\{\operatorname{dim} \mathcal{M}_{y} \mid y \in Y\right\}$.

(5.11) Theorem. (1) For $(y \in Y, t \in T) \operatorname{dim} \mu_{y}=\operatorname{dim} M_{y t}$, and

(2) the set $L=\left\{y \mid \operatorname{dim} M_{y}=r\right\}$ is an open, dense, T-invariant subset of $Y$.

Proof. (1) Since $\mathcal{M}$ is $T$-invariant, the map $\alpha \rightarrow \alpha t: \underline{U} \rightarrow \underline{U}$ induces an isomorphism of $\mathcal{M}_{y}$ onto $\mathcal{M}_{y}$. 
(2) Let $y \in L, h^{1}, \ldots, h^{r} \in \mathcal{M}$ such that $h_{y}^{1}, \ldots, h_{y}^{r}$ are independent. Then $\operatorname{det}\left(\left(h^{i} \mid h^{j}\right) z\right) \neq 0$ for all $z$ in some neighbourhood $N$ of $y$. Consequently $\operatorname{dim} \mathcal{M}_{z}=r$ $(z \in N)$ and so $L$ is open. It is $T$-invariant by (1) whence $\bar{L}=Y$ since $Y$ is ergodic and $\bar{L}$ is both open and closed. (Recall that $Y$ is extremely disconnected.)

(5.12) TheOREM. There exists an open dense subset $K$ of $Y$ and $\sigma_{1}, \ldots, \sigma_{r} \in \Gamma(K, \mathcal{M})$ such that $\left(\sigma_{i}(y) \mid \sigma_{j}(y)\right)_{y}=\delta_{i j}(y \in Y)$.

Proof. Let $L$ be as in (5.11). Then the Gram-Schmidt orthonormalization process shows that for each $y \in Y$ there exists a neighbourhood $N$ of $y$ and $\rho^{1}, \ldots, \rho^{r} \in$ $\Gamma(N, \mathcal{M})$ with $\left(\rho^{i}(z) \mid \rho^{j}(z)\right)_{z}=\delta_{i j}(z \in N)$. An application of Zorn's lemma now gives (5.12).

(5.13) ThEOREM. Let $K, \sigma_{1}, \ldots, \sigma_{r}$ be as in (5.12), $S=\Pi^{-1}(K), \psi: S \rightarrow \mathbb{C}^{r}, \varphi: S \rightarrow \mathbb{R}$ such that $\psi(x)=\left(\sigma_{1} \Pi(x)(x), \ldots, \sigma_{r} \Pi(x)(x)\right)$ and $\varphi(x)=\|\psi(x)\|(x \in S)$. Then $\varphi$ is a constant not equal to zero.

Proof. By (5.4) $\psi$ is continuous, whence so is $\varphi$. Now let $x, x t \in S, y=\Pi x$. Then

$$
\sigma_{i}(y)(t)=\sum_{j=1}^{r} A_{i}^{j}(y, t) \sigma_{i}(y t)
$$

$(1 \leq i \leq r)$ with $\left(A_{i}^{j}\right)$ unitary since the map $t: \mathscr{U}_{y} \rightarrow \mathscr{U}_{y t}$ preserves the inner product.

Now by (5.5) there exist $g^{1}, \ldots, g^{r} \in \mathcal{U}$ such that $\sigma_{i}(y)=g_{y}^{1}$ and $\sigma_{i}(y t)=g_{y t}^{i}$ $(1 \leq i \leq r)$. Then $\psi(x)=\left(g^{1}(x), \ldots, g^{r}(x)\right)$ and $\psi(x t)=\left(g^{1}(x t), \ldots, g^{r}(x t)\right)$ and $(*)$ becomes

$$
\left(t^{-1} g^{i}\right)_{y t}=\sum_{j} A_{i}^{j}(y, t) g_{y t}^{j}
$$

whence $g^{i}(x)=\sum_{j} A_{i}^{J}(y, t) g^{j}(x t)$ and so $\varphi(x)=\|\psi(x)\|=\|\psi(x t)\|=\varphi(x t)$ since $\left(A_{i}^{j}\right)$ is unitary.

Thus $t \varphi=\varphi$ on $S \cap S t^{-1}$. Since $m(S)=m_{Y}(K)=1, m\left(S \cap s t^{-1}\right)=1$ whence $t \varphi=\varphi$ (a.e. $m(t \in T)$ ). The ergodicity of $m$ now implies that $\varphi$ is a constant. This constant cannot be zero since $\sigma_{1}(y), \ldots, \sigma_{r}(y)$ is a basis for $\mathcal{M}_{y}(y \in K)$.

(5.14) THEOREM. With the same notation as in (5.12) there exist $g^{1}, \ldots, g^{r} \in \mathcal{U}$ such that $g_{y}^{i}=\sigma_{y}(1 \leq i \leq r, y \in K)$.

Proof. By (5.13) there exists $c>0$ such that $\psi(x)=\left(\sigma_{1}\left(\Pi x(x), \ldots, \sigma_{r}(\Pi x)(x)\right)\right.$ is a continuous map of the dense open subset $S$ of $U$ into the sphere of radius $c$ in $\mathbb{C}^{r}$. Since the latter is compact there is a continuous extension $\bar{\psi}$ of $\psi$ to all of $U$ [2]. The $r$ components, $g^{1}, \ldots, g^{r}$ of $\bar{\psi}$ then satisfy the relation $g_{y}^{1}=\sigma_{i}(y)(1 \leq i \leq r$, $y \in K$ ).

(5.15) Theorem. Let $g^{1}, \ldots, g^{r}, h^{1}, \ldots, h^{r} \in \mathcal{U}$ be such that $\left(g_{y}^{i} \mid g_{y}^{j}\right)=\delta_{i j}=\left(h_{y}^{i} \mid h_{y}^{j}\right)$ $(1 \leq i, j \leq r, y \in Y)$ and $\operatorname{span}\left\{g_{y}^{i} \mid 1 \leq i \leq r\right\}=\mu_{y}=\operatorname{span}\left\{h_{y}^{i} \mid 1 \leq i \leq r\right\}$ for all $y$ in $a$ dense subset $S$ of $Y$, and let $\mathscr{L}=\mathscr{F} g^{1}+\cdots+\mathscr{F} g^{r}, \mathcal{N}=\mathscr{F} h^{1}+\cdots+\mathscr{F} h^{r}$. Then (i) $\mu \subset \mathcal{N} \cap \mathscr{L}$; (ii) $\mathcal{N}=\mathscr{L}$, (iii) $\mathscr{L}$ is $T$-invariant.

Proof. (i) Let $f \in \mathcal{M}$. Then $f_{y}=\sum_{i=1}^{n} c_{i}^{y} g_{y}^{i}(y \in S)$ whence

$$
f(x)=\sum_{i} c_{i}^{\Pi 1 x}(x) g^{i}(x) \quad\left(x \in \Pi^{-1}(S)\right)
$$


Since $c_{i}^{\Pi x}(x)=\left(f_{\Pi x} \mid g_{\Pi x}^{i}\right)(x)$ is continuous and $\Pi^{-1}(S)$ is dense (recall that $\Pi$ is open), (*) holds for all $x \in U$, whence $f \in \mathscr{F} g^{1}+\cdots+\mathscr{F} g^{r}=\mathscr{L}$. Similarly $f \in \mathcal{N}$.

(ii) If $f \in \mathcal{N}$, the argument given above shows that $f \in \mathscr{L}$. Similarly $\mathscr{L} \subset \mathcal{N}$.

(iii) Let $f \in \mathscr{L}, t \in T$. Then $(t f)_{y}=\left(f_{y t}\right) t^{-1},(y \in Y)$, and if $y \in S \cap S t^{-1}$,

$$
\mathscr{L}_{y t} t^{-1}=\mathscr{M}_{y t} t^{-1}=M_{y}=\mathscr{L}_{y}
$$

whence $(t f)_{y}=\sum_{i} d_{i}^{y} g_{y}^{i}$ and one proceeds as before to show that $t f \in \mathscr{L}$.

(5.16) Remarks. (1) Theorems (5.14) and (5.15) show that there is a well defined $T$-invariant, $\mathscr{F}$-submodule, $\hat{\mathcal{M}}$ of $\mathcal{U}$ such that $\mathcal{M} \subset \hat{\mathscr{M}}$ and there exist $g^{1}, \ldots, g^{\prime} \in \hat{\mathcal{M}}$ with $\left(g_{y}^{i} \mid g_{y}^{j}\right)=\delta_{i j}$ and $\hat{M}_{y}=\operatorname{span}\left\{g_{y}^{1}, \ldots, g_{y}^{r}\right\}(1 \leq i, j \leq r ; y \in Y)$.

(2) If $\mathscr{A}$ is a $T$-invariant, conjugate closed subalgebra of $\mathcal{U}$ with $M \subset \mathscr{A}$ and $\mathscr{A}=\tilde{A}$, then $\hat{\mathcal{M}} \subset \mathscr{A}$. (To see this observe that all the results go through with $\mathcal{U}$ replaced by $\mathscr{A}$.)

(5.17) Definition. The goal is now to construct an almost periodic extension of $Y$ using the module, $\hat{\mathcal{M}}$. (Compare chapter 17 of [3].)

To this end set $X_{y}=\left\{x \mid x: \mathbb{C}^{r} \rightarrow \hat{M}_{y}\right.$ an isometry $\}, X$ the disjoint union, $+\left\{X_{y} \mid y \in Y\right\}$ and $\rho: X \rightarrow Y$ the canonical map.

Let $t \in T, x \in X_{y}$. Since $\alpha \rightarrow \alpha t: \hat{\mathcal{M}}_{y} \rightarrow \hat{\mathcal{M}}_{y t}$ is an isometry the composite map $x t(v)=$ $x(v) t\left(v \in \mathbb{C}^{r}\right)$ is in $X_{y t}$, and the map $(x, t) \rightarrow x t: X \times T \rightarrow X$ defines an action of $T$ on $X$.

Let $G$ be the unitary group, $U(r)$. Then the map $(\alpha, x) \rightarrow \alpha x: G \times X \rightarrow X$ defines a free action of $G$ on $X$. Here $(\alpha x)(v)=x\left(\alpha^{-1}(v)\left(x \in X, \alpha \in G, v \in \mathbb{C}^{r}\right)\right.$.

Moreover the action of $G$ commutes with that of $T$ so that $(G, X, T)$ is a bitransformation group.

For $g \in \hat{M}, v \in \mathbb{C}^{r}$ let $\langle g, v\rangle: X \rightarrow \mathbb{C}$ be such that

$$
\langle g, v\rangle(x)=\left(x(v) \mid g_{\rho(x)}\right)_{\rho(x)} .
$$

Finally let $\mathscr{T}$ be the smallest topology on $X$ making the maps $\langle g, v\rangle$ and $x \rightarrow f(\rho(x))$ : $X \rightarrow \mathbb{C}$ continuous $\left(g \in \hat{M}, v \in \mathbb{C}^{r}, f \in \mathscr{F}\right)$.

(5.18) TheOrem. (1) The topology $\mathscr{T}$ is Hausdorff.

(2) The map $\rho: X \rightarrow Y$ is continuous.

(3) The map $y \rightarrow^{\sigma} e_{y}: Y \rightarrow X$ is continuous, where $e_{y}$ is the linear map of $\mathbb{C}^{r}$ into $X_{y}$ which maps the canonical basis vector $e_{i}$ of $\mathbb{C}^{r}$ onto $g_{y}^{i}(1 \leq i \leq r)$.

(4) The map $(\alpha, x) \rightarrow^{L} \alpha x: G \times X \rightarrow X$ is continuous.

(5) The map $(x, t) \rightarrow{ }^{B} x t: X \times T \rightarrow X$ is continuous.

(6) $(X, \mathscr{T})$ is compact.

Proof. (1) The set $\left\{\langle g, v\rangle \mid g \in \hat{M}, v \in \mathbb{C}^{r}\right\} \cup\{f \circ \rho \mid f \in \mathscr{F}\}$ separates points of $X$.

(2) The maps $\{f \circ \rho \mid f \in \mathscr{F}=C(Y)\}$ are continuous.

(3) Let $f \in \mathscr{F}$. Then $(f \circ \rho) \circ \sigma=f$ which is continuous.

Now let $g \in \hat{M}, v \in \mathbb{C}^{r}$. Then $v=\sum v^{i} e_{i}$ whence

$$
(\langle\mathrm{g}, v\rangle \circ \sigma)(y)=\left(\sigma(y)(v) \mid g_{y}\right)=\sum v^{i}\left(g^{i} \mid g\right)_{y}
$$


Then $\langle g, v\rangle \circ \sigma$ is continuous and so $\sigma$ is continuous by the definition of $\mathscr{T}$.

(4) Let $f \in \mathscr{F}$. Then

$$
f \circ \rho \circ L(\alpha, x)=f(\rho(\alpha x))=f(\rho(x)) .
$$

Hence $f \circ \rho \circ L$ is continuous.

Let $g \in \hat{M}, v \in \mathbb{C}^{r}$. Then

$$
\begin{aligned}
\langle g, v\rangle(L(\alpha, x)) & =\langle g, v\rangle(\alpha x)=\left(x\left(\alpha^{-1} v\right) \mid g_{\rho(x)}\right) \\
& =\sum_{i} a^{i}(\alpha, v)\left(x\left(e_{i}\right) \mid g_{\rho(x)}\right)=\sum a^{i}(\alpha, v)\left\langle g_{i} \mid e_{i}\right\rangle(x),
\end{aligned}
$$

where $\alpha^{-1} v=\sum_{i} a^{i}(\alpha, v) e_{i}$.

Thus $\langle g, v\rangle \circ L$ is continuous. Hence $L$ is continuous.

(5) Let $f \in \mathscr{F}, t \in T$. Then $f \circ \rho(x t)=(t f \circ \rho)(x)$ whence $f \circ \rho \circ R_{t}$ is continuous since $t f \in \mathscr{F}$. (Here $R_{t}(x)=x t(x \in X)$.)

Let $g \in \hat{M}, v \in \mathbb{C}^{r}$. Then

$$
\langle g, v\rangle(x t)=\left((x t)(v) \mid g_{y t}\right)=\left(x(v) t \mid g_{y t}\right)=\left(x(v) \mid(t g)_{y}\right)
$$

(where $y=\rho(x)$ ).

Thus $R_{t}$ is continuous ( $t \in T$ ). Hence $R$ is continuous since $T$ is discrete.

(6) This follows from (3), (4) and the fact that $X=L(G \times \sigma(Y))$.

(5.19) Notation. For the remainder of this section the following notation will be in force: $S$ the sphere of radius $c=\|\psi(x)\|(x \in X)$ (see (5.13)), $S \times{ }_{G} X$ the orbit space $S \times X / G$ where $\alpha(v, x)=(\alpha v, \alpha x)(\alpha \in G, v \in S, x \in X)$, and $[v, x]$ the image of $(v, x)$ under the canonical map of $S \times X$ into $S \times{ }_{G} X$.

Let $T$ act on $S \times X$ via the map $(s, x, t) \rightarrow(s, x t): S \times X \times T \rightarrow S \times X$. Then $(G$, $S \times X, T)$ is a bitransformation group and so there is induced an action of $T$ on $S \times{ }_{G} X$.

(5.20) Theorem. The map $u \rightarrow_{\gamma}[h(u), \sigma \Pi(u)]: U \rightarrow S \times_{G} X$ is a homomorphism; where $h(u)=\left(g^{1}(u), \ldots, g^{r}(u)(u \in U)\right.$.

Proof. Let $u \in U, t \in T$ and $y=\Pi(u)$. Since $\sigma(y) t$ and $\sigma(y t)$ are both isometries of $\mathbb{C}^{r}$ onto $\hat{M}_{y t}$, there exists $\beta \in G$ with $\sigma(y t) \circ \beta=\sigma(y) t$; i.e. $\beta^{-1} \sigma(y t)=\sigma(y) t$.

If $\beta\left(e_{i}\right)=\sum_{j=1}^{r} \beta_{j i} e_{j}$ then this says that $t^{-1} g_{y}^{i}=\sum_{j} \beta_{j i} g_{y t}^{j}$ whence

$$
g^{i}(u)=\sum_{j} \beta_{j i} g^{j}(u t)=\sum\left(\beta^{-1}\right)_{i j} g^{j}(u t)
$$

$(1 \leq i \leq r)$. Consequently $\beta^{-1}(h(u t), \sigma(y t))=(h(u), \sigma(y) t)$ and so

$$
\begin{aligned}
\gamma(u t) & =[h(u t), \sigma \Pi(u t)]=[h(u t), \sigma(y t)] \\
& =[h(u), \sigma(y) t]=[h(u), \sigma(y)] t=\gamma(u) t .
\end{aligned}
$$

The proof is complete.

(5.31) Remark. As in (4.9) the section $\sigma$ gives rise to cocycle $\delta$ on $Y \times T$ to $G$ such that the map $(\alpha, y) \rightarrow{ }_{\psi} \alpha \sigma(y): G \times Y \rightarrow X$ is a bitransformation group isomorphism. (Recall $\delta(y, t) \sigma(y t)=\sigma(y) t(y \in Y, t \in T$.) The cocycle $\delta$ may be used to define an action of $T$ on $S \times Y$ viz:

$$
(v, y) t=\left(\delta(y, t)^{-1} v, y t\right) \quad(v \in S, y \in Y, t \in T) .
$$


Denote this flow by $S \times{ }_{\delta} Y$. Now it is easy to check that the diagram

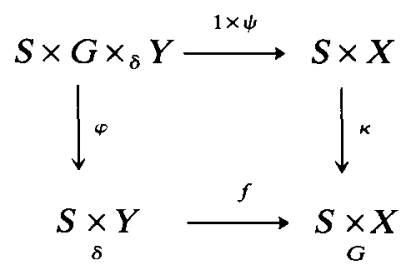

is commutative and that $f$ is an isomorphism. Here $\varphi(v, \alpha, y)=\left(\alpha^{-1}(v), y\right)$ $(v \in S, \alpha \in G, y \in Y), \kappa$ is the canonical map and $f(v, y)=[v, \sigma(y)](v \in S, y \in Y)$.

(5.32) Notation. In addition to the other assumptions made in this section the following will be in force: $\mathscr{A}$ will denote a $T$-invariant subalgebra of $U$ with $\mathscr{F} \subset \mathscr{A}=\dot{A}$ and $\underline{M}$ the set of finitely generated $T$-F submodules $\mathcal{M}$ of $\mathcal{U}$ with $\mathscr{F} \subset M \subset \mathscr{A}$.

(5.33) LemMA. Let $\mathcal{M} \in \underline{M}$. Then there exists a compact group $K$, a cocycle $\eta$ on $Y \times T$ to $K$, a closed subgroup $H$ of $K$ and a $T$-subalgebra $\mathscr{B}$ of $\mathscr{A}$ with $\mathscr{M} \subset \mathscr{B}$ and $|\mathscr{B}|$ isomorphic to $\mathrm{K} / \mathrm{H} \times{ }_{\eta} \mathrm{Y}$.

Proof. As in (5.31) we shall identify $G \times{ }_{\delta} Y$ with $X$ and $S \times{ }_{\delta} Y$ with $S \times{ }_{G} X$. The assumptions on $\mathscr{A}$ suffice to justify replacing $U$ by $|\mathscr{A}|$ in (5.20). This provides a homeomorphism $\gamma$ of $|\mathscr{A}|$ into $S \times{ }_{\delta} Y$.

Let $s_{0}$ be a fixed element of $S, \psi: G \times_{\delta} Y \rightarrow S \times{ }_{\delta} Y$ be such that $\psi(\alpha, y)=\left(\alpha\left(s_{0}\right), y\right)$ $(\alpha \in G, y \in Y), \varphi: S \times_{\delta} Y \rightarrow Y$ be such that $\varphi(s, y)=y$, and $W=\operatorname{im} \gamma$. Then $\psi, \varphi$ are epimorphisms and $\varphi \psi$ is the projection onto $Y$.

Now let $\nu=m_{s \&} \gamma^{-1}$. Then $\nu$ is an ergodic, $T$-invariant supported measure on $W$ such that $m=\nu \varphi^{-1}$ (notice that $\varphi \circ \gamma$ is the canonical map of $|\mathscr{A}|$ onto $Y$.).

The measure $\nu$ on $W \subset S \times{ }_{\delta} Y$ can be lifted to a $T$-invariant measure on $G \times{ }_{\delta} Y$ since the latter is a compact group extension of $S \times{ }_{\delta} Y$.

The preceding paragraphs verify the conditions of theorem (4.16) with $G \times{ }_{\delta} Y=X$, $S \times{ }_{\delta} Y=E$ and the other symbols identical. Lemma (5.3) now follows from (4.16).

(5.34) Remark. With the notation as in (5.33) $C\left(E / H \times_{\delta} Y\right)=\mathscr{B} \subset \mathscr{A}$. In general $C\left(E \times_{\delta} Y\right)$ need not be contained in $\mathscr{A}$. However by enlarging $\mathcal{U}$ if necessary we may (and do) assume that $C\left(E \times{ }_{\delta} Y\right) \subset \mathscr{U}$ for all such group extensions.

(5.35) LEMMA. Let $\left(\mathscr{C}_{i} \mid i \in I\right)$ be a family of $T$-subalgebras of $\mathcal{U}$ such that $\left|\mathscr{C}_{i}\right|$ is a group extension of $\mathscr{F}(i \in I)$. Then $\left|V \mathscr{C}_{i}\right|$ is also a group extension of $\mathscr{F}$.

Proof. Let $G_{i}$ be a compact group, $\delta_{i}$ a cocycle on $Y \times T$ to $G_{i}, \Pi_{j}:\left|\mathscr{C}_{i}\right| \rightarrow Y$ the canonical map and $\psi_{i}:\left|\mathscr{C}_{i}\right| \rightarrow G_{i}$ such that $\psi_{i} \times \Pi_{i}$ is an isomorphism of $\left|\mathscr{C}_{i}\right|$ onto $G_{i} \times_{\delta_{i}} Y(i \in I)$.

Set $G=\Pi_{i} G_{i}$ and $\delta(y, t)=\left(\delta_{i}(y, t) \mid i \in I\right)$. Then $\delta$ is a cocycle on $Y \times T$ to $G$. Let $\kappa_{i}: U \rightarrow\left|\mathscr{C}_{i}\right|(i \in I)$ and $\kappa: U \rightarrow Y$ be the canonical maps and $\varphi: U \rightarrow G \times{ }_{\delta} Y$ be such that $\varphi(u)=\left(\left(\psi_{i} \kappa_{i} u \mid i \in I\right), \kappa(u)\right)(u \in U)$.

Then it is immediate that $\varphi$ is a homeomorphism, $\varphi(U)$ projects onto $Y$, and that $m \varphi^{-1}$ is a $T$-invariant ergodic measure supported on $\varphi(U)$. Consequently by the results of $\S 4, \varphi(U)$ is a compact group extension of $Y$. 
Since $\varphi(u)=\varphi(v)$ if and only if $\kappa_{i}(u)=\kappa_{i}(v)(i \in I), \varphi^{*} C(\varphi(U))=\bigvee \mathscr{C}_{i}$. The proof is complete.

(5.36) TheOREм. Let $\mathscr{A}$ be the smallest $\mathscr{T}_{m}$ closed $T$-subalgebra of $U$ containing $\bigcup\{\mathcal{M} \mid \mathcal{M} \in \underline{M}\}$. Then there exists a T-subalgebra $\mathscr{L}$, a compact group $G$, a closed subgroup $S$ of $G$ and a cocycle $\rho$ on $Y \times T$ to $G$ such that $|\mathscr{L}| \cong G / S \times Y$ and $\tilde{\mathscr{L}}=\mathscr{A}$.

Proof. For each $\mathcal{M} \in \mathscr{M}$ let $\mathscr{B}(\mathcal{M})$ be as in (5.33) and $\mathscr{C}(\mathcal{M})$ the corresponding group extension $C\left(E \times{ }_{\delta} Y\right)$. Then $\bigvee \mathscr{C}(\mathcal{M})$ is a group extension of $Y$ by (5.35) and it is immediate that

$$
|V \mathscr{B}(\mathcal{M})| \cong G / S \underset{\rho}{\times} Y
$$

for some closed subgroup $S$ of $G$ where $|\bigvee \mathscr{C}(\mathcal{M})| \cong \mathrm{G} \times_{\mathrm{p}} Y$.

Set $\mathscr{L}=\bigvee \mathscr{B}(\mathscr{M})$. Then $\mathcal{M} \subset \mathscr{L} \subset \tilde{\mathscr{L}} \subset \mathscr{A}(\mathscr{M} \in \underline{M})$ implies that $\tilde{\mathscr{L}}=\mathscr{A}$.

\section{REFERENCES}

[1] N. Bourbaki. Intégration. Hermann, Paris, 1965.

[2] J. Dixmier. Sur certains espaces considérés par. M. H. Stone. Summa Brasil, Math. t.2. fasc. 11 (1951), 151-182.

[3] R. Ellis. Locally compact transformation groups. Duke Math. Journal, 24 (1957), 119-125.

[4] R. Ellis. Lectures on Topological Dynamics. Benjamin, New York, 1969.

[5] R. Ellis. The Furstenberg structure theorem. Pac. J. Math. 76 (1978), 345-349.

[6] A. M. Gleason. Projective topological spaces, Ill. J. Math. 2 (1958), 482-489.

[7] K. H. Hofmann \& K. Keimel. Bundles and sheaves of Banach spaces, C(X)-modules. Lec. Notes in Math. 753 (Berlin, Springer-Verlag) (1979), 415-441.

[8] H. B. Keynes \& D. Newton. The structure of ergodic measures for compact group extensions. Israel J. of Math., 18 (1974), 363-399.

[9] G. W. Mackey. Point realizations of transformation groups. Ill. J. Math., 6 (1962), 327-335.

[10] R. J. Zimmer. Extensions of ergodic group actions. Ill. J. Math. 20 (1976), 373-409. 